hep-ph/0504082

OSU-HEP-05-05

April, 2005

\title{
Coupling Unifications in Gauge-Higgs Unified Orbifold Models
}

\author{
Ilia Gogoladze ${ }^{a 1}$, Tianjun Li ${ }^{b 2}$, Yukihiro Mimura ${ }^{c 3}$ and S. Nandi ${ }^{d 4}$ \\ ${ }^{a}$ Department of Physics, University of Notre Dame, Notre Dame, IN 46556, USA \\ ${ }^{b}$ School of Natural Sciences, Institute for Advanced Study, \\ Einstein Drive, Princeton, NJ 08540, USA \\ ${ }^{c}$ Department of Physics, University of Regina, Regina, Saskatchewan S4S 0A2, Canada \\ ${ }^{d}$ Physics Department, Oklahoma State University, Stillwater, OK 74078 USA
}

\begin{abstract}
Supersymmetric gauge theories, in higher dimensions compactified in an orbifold, give a natural framework to unify the gauge bosons, Higgs fields and even the matter fields in a single multiplet of the unifying gauge symmetry. The extra dimensions and the supersymmetry are the two key ingredients for such an unification. In this work, we investigate various scenarios for the unification of the three gauge couplings, and the Yukawa couplings in the Minimal Supersymmetric Standard Model (MSSM), as well as the trilinear Higgs couplings $\lambda$ and $\kappa$ of the Non-Minimal Supersymmetric Standard Model (NMSSM). We present an $S U(8)$ model in six dimensions with $N=2$ supersymmetry, compactified in a $T^{2} / Z_{6}$ orbifold which unifies the three gauge couplings with $\lambda$ and $\kappa$ of NMSSM. Then, we present an $S U(9)$ model in 6D, which, in addition, includes partial unification of Yukawa couplings, either for the up-type (top quark and Dirac tau-neutrino) or down-type (bottom quark and tau lepton). We also study the phenomenological implications of these various unification scenarios using the appropriate renormalization group equations, and show that such unification works very well with the measured low energy values of the couplings. The predicted upper bounds for the lightest neutral Higgs boson mass in our model is higher than those in MSSM, but lower that those in the general NMSSM (where the couplings $\lambda$ and $\kappa$ are arbitrary). Some of the predictions of our models can be tested in the upcoming Large Hadron Collider.
\end{abstract}

\footnotetext{
${ }^{1}$ email: gogoladze.1@nd.edu

2email: tli@ias.edu

${ }^{3}$ email: mimura2y@uregina.ca

${ }^{4}$ email: shaown@okstate.edu
} 


\section{Introduction}

The Standard Model (SM) is well established to describe the physics below the weak scale. The key ingredient of the electroweak theory is the Higgs mechanism, in which the electroweak gauge symmetry, $S U(2)_{L} \times U(1)_{Y}$, is spontaneously broken down to the electromagnetic gauge symmetry, $U(1)_{E M}$, by the vacuum expectation values (VEVs) of Higgs doublets. The VEVs of Higgs doublets not only do make the $W$ and $Z$ bosons massive, but also give masses to the quarks and leptons through Yukawa couplings. Although the Higgs bosons have not been observed yet, it is expected that there is rich physics in the Higgs sector which will be tested at the upcoming colliders. The elementary particles, which we have already observed, are the SM fermions and gauge bosons, while the Higgs particles are scalar fields. The radiative corrections to the Higgs boson (or scalars in general) masses are quadratically dependent on the UV cutoff scale $\Lambda$, and are not protected by chiral or gauge symmetries. Thus, the natural Higgs masses are of order $\Lambda$ rather than the weak scale, leading to the gauge hierarchy problem because $\Lambda$ should be around the Planck or string scale. It is expected that there exists new physics around a TeV scale from naturalness of the Higgs masses. A well-known solution to the gauge hierarchy problem is supersymmetry. In supersymmetric theories, each particle has a superpartner which differs in spin by $1 / 2$ and is related to the original particle by a supersymmetry transformation. Since supersymmetry relates the scalar and fermionic sectors, the chiral symmetries, which protect the fermion masses, also protect the scalar masses against the quadratic divergences, leading to an elegant solution to the gauge hierarchy problem. As a result, there are only logarithmic divergences in the scalar masses and couplings in supersymmetric theories and they can be extrapolated to the higher energy using the evolution of the Renormalization Group Equations (RGEs). In fact, in the Minimal Supersymmetric Standard Model (MSSM), the three gauge couplings in the SM are unified at a scale of about $2 \times 10^{16} \mathrm{GeV}$ [1], which implies a possible Grand Unified Theory (GUT). In addition to the gauge coupling unification, the Yukawa couplings can be unified (bottom-tau [2, top-bottom-tau [3], or other types of Yukawa unification [4]). In such a way, the supersymmetric theories are suitable to connect the weak scale physics to the high scale physics in the unified pictures.

Recently, the gauge-Higgs unification [5, 6, 7] in higher-dimensional models is considered as one of the theoretical origins of Higgs bosons. Since the extra-dimensional components of gauge fields transform as scalar fields in four dimension (4D), the zero modes of the extra components can be identified as Higgs fields. The Higgs fields, which break the electroweak gauge symmetry, are the doublets under $S U(2)_{L}$ while the gauge fields are in the adjoint representation, and thus one needs to extend the electroweak gauge group in order to realize the gauge-Higgs unification. The gauge symmetry is broken by the orbifold boundary condition [8, 9], and the remaining 
electroweak gauge symmetry is broken further by the Higgs multiplets which are the zero modes for the broken generators of the bulk gauge group. This is the well-known Hosotani mechanism [6]. Therefore, the gauge-Higgs unification is compatible with the gauge symmetry unification in orbifold GUTs [10]. Furthermore, if one realizes the gauge-Higgs unification, the Yukawa couplings may arise from the gauge interaction since the covariant derivative in higherdimensional kinetic term, $\bar{\Psi} \Gamma^{M} A_{M} \Psi$, includes Yukawa couplings. The realistic models in gaugeYukawa unification are constructed in 5-dimensional (5D) supersymmetric models [11, 12]. In 6-dimensional (6D) models, the quark and lepton fields can also be unified with the gauge multiplet in higher dimensions [13, 14. In fact, the unification of gauge and Yukawa couplings for quarks and leptons in third generation are in very good agreement with experiments [15], which gives us a beautiful unified picture at compactification scale.

Although the supersymmetric model can explain the gauge hierarchy problem naturally, the supersymmetric Higgs mass $\mu$ need not be hierarchically small compared to the Planck scale or string scale because it is allowed by gauge symmetry and supersymmetry. To break the electroweak gauge symmetry, the $\mu$-term (and the $B$-term, which is a supersymmetry breaking Higgs bilinear soft mass term) must be close to the weak scale and the supersymmetry breaking scale. Thus, how to explain the weak scale $\mu$-term is an interesting question. This is known as the $\mu$-problem. One usually assumes a symmetry to suppress the $\mu$-term [16]. For example, the Giudice-Masiero mechanism [17] can solve the $\mu$-problem in the supergravity mediated supersymmetry breaking scenario because the $\mu$-term is forbidden by the $R$ symmetry and is generated after the supersymmetry breaking. An alternative solution to the $\mu$-problem is the Next to the MSSM [18] in which a singlet field $S$ and the superpotential $\lambda S H_{u} H_{d}$ are introduced. The explicit $\mu$-term is forbidden by the discrete $Z_{3}$ symmetry, and the effective $\mu$-term $\mu=$ $\lambda\langle S\rangle$ is generated after $S$ obtains a VEV. Thus, $\mu$ is naturally around the supersymmetry breaking scale due to the minimization condition of the scalar potential. This type of model is easily realized in the context of supersymmetric gauge-Higgs unification when the bulk gauge symmetry is appropriately extended and the singlet can be obtained as a massless mode from the bulk gauge multiplet [19]. The bulk gauge multiplet can naturally include two extra singlet fields $S_{1}$ and $S_{2}$, and the $\kappa S S_{1} S_{2}$ coupling can come from the bulk interaction in addition to the coupling $\lambda S H_{u} H_{d}$. Thus, the $\lambda$ and $\kappa$ couplings can be unified with the electroweak gauge couplings at the compactification scale.

In this paper, we study various scenarios of coupling unification in the gauge-Higgs unification, and gauge-Higgs-matter unification where in addition to the Higgs trilinear couplings $\lambda$ and $\kappa$, we also have the unification of the Yukawa couplings for quarks and leptons in the third generation. The gauge, Higgs and Yukawa couplings can be unified at compactification scale. 
Depending on the bulk gauge group and the hypercharge assignments, the up-type (top quark and tau-neutrino Dirac) Yukawa couplings or the down-type (bottom quark and tau) Yukawa couplings are unified with the gauge couplings. Especially, the unification of the gauge, Higgs trilinear and top Yukawa couplings is an interesting possibility since it can explain why the top quark is the heaviest fermion (for the small $\tan \beta$ case). We also study the numerical predictions of the couplings at low energy. We will show that the prediction of the Yukawa coupling unification is in good agreement with experiments, in particular, the top quark mass prediction is an important result of the gauge-Higgs-Yukawa coupling unification at the compactification scale.

This paper is organized as follows: In Section 2, we study what bulk gauge symmetries realizing the gauge-Higgs unification with gauge and Yukawa coupling unification. The NMSSM superpotential can be generated from the bulk gauge interaction when the appropriate bulk gauge symmetries are considered. In Sections 3 and 4, we will construct $6 \mathrm{D} S U(8)$ and $S U(9)$ supersymmetric models on $T^{2} / Z_{6}$ orbifold. In Section 5, we propose 7-dimensional (7D) $S U(9)$ models on $T^{2} / Z_{6} \times S^{1} / Z_{2}$ orbifold. In Section 6, we present the numerical results of the gauge, Yukawa and/or Higgs trilinear coupling unifications. Section 7 contains our discussions and conclusions. In Appendix A, we study the gauge-Higgs unification in 6D supersymmetric models on $T^{2} / Z_{6}$ orbifold.

\section{Choice of Bulk Gauge Symmetry}

The $\mathcal{N}=1$ supersymmetry in $5 \mathrm{D}$ corresponds to $\mathcal{N}=2$ supersymmetry in $4 \mathrm{D}$, and the Higgs fields can be contained in $\mathcal{N}=2$ gauge multiplet [7, 11]. Matter fields are contained in the hypermultiplets. On the other hand, $\mathcal{N}=(1,1)$ supersymmetry in $6 \mathrm{D}$ corresponds to $\mathcal{N}=4$ supersymmetry in $4 \mathrm{D}$, and thus the models are restricted since only the gauge multiplet can be introduced in the bulk. In terms of $4 \mathrm{D} \mathcal{N}=1$ supersymmetry language, the $6 \mathrm{D}$ gauge multiplet contains vector multiplet $V\left(A_{\mu}, \lambda\right)$ and three chiral multiplet, $\Sigma_{i}(i=1,2,3)$, in adjoint representation of gauge group. In a sense, the 5D hypermultiplets in the adjoint representation can also belong to gauge multiplet, and thus one can consider that the matter representation also belong to the bulk gauge multiplets [13.

In this Section, we will study what bulk gauge symmetry can realize the gauge-Higgs unification with various coupling unifications. 


\subsection{Gauge-Higgs Unification and Yukawa Couplings}

The minimal choice of bulk gauge symmetry to realize the gauge-Higgs unification is $S U(3)_{W}$ [11, 20]. The bulk symmetry is broken as $S U(3)_{W} \rightarrow S U(2)_{L} \times U(1)_{Y}$, and the Higgs doublets $\left(H_{u}\right.$ and $\left.H_{d}\right)$ are included in the gauge multiplet, $\mathbf{8}=\mathbf{3}_{0}+\mathbf{2}_{1 / 2}+\mathbf{2}_{-1 / 2}+\mathbf{1}_{0}$. The hypercharge generator is $T_{Y}=\operatorname{diag}(1,1,-2) / 6$, and thus the predicted weak mixing angle at compactification scale is $\sin ^{2} \theta_{W}=3 / 4$, which is too large for usual minimal unification pictures through RGEs. One needs to add extra fields to change the RGE evolution or to consider that the brane localized gauge couplings give the dominant contributions to the 4D gauge couplings rather than the bulk gauge coupling.

In order to realize the proper weak mixing angle in the minimal unification scenario, $\sin ^{2} \theta_{W}=3 / 8$, we should consider $S U(4)_{W}$ bulk gauge symmetry rather than $S U(3)_{W}$. The $S U(4)_{W}$ adjoint representation, 15, is decomposed as $\mathbf{1 5}=(\mathbf{3}, \mathbf{1})_{0}+(\mathbf{1}, \mathbf{3})_{0}+(\mathbf{2}, \mathbf{2})_{2}+(\mathbf{2}, \mathbf{2})_{-2}+$ $(\mathbf{1}, \mathbf{1})_{0}$ under $S U(4)_{W} \rightarrow S U(2)_{L} \times S U(2)_{R} \times U(1)^{\prime}$, and it is easy to see that the Higgs bidoublets are included in the bulk gauge multiplet. Thus, considering $S U(3)_{c} \times U(1)_{B-L} \times S U(4)_{W}$ bulk symmetry, we can obtain the proper weak mixing angle [12, 21]. For this, the leftright symmetric base (or Pati-Salam base [22]) is useful because the Higgs bidoublets are not charged under the $U(1)_{B-L}$. One can also realize the same quantum number assignment in $S O(5)_{W}\left(\simeq S p(4)_{W}\right)$ as a minimal choice: $S O(5)$ adjoint $\mathbf{1 0}=(\mathbf{3}, \mathbf{1})+(\mathbf{1}, \mathbf{3})+(\mathbf{2}, \mathbf{2})$ under $S O(5)_{W} \rightarrow S U(2)_{L} \times S U(2)_{R}$. Of course, in such product bulk gauge symmetries, the $B-L$ charge normalization is arbitrary and thus the weak mixing angle is not completely determined. One can unify the bulk gauge groups into simple groups, $S O(12)(S O(11))$ or $S U(8)$. One can also consider $E_{6}$ as a GUT group using $E_{6} \rightarrow S U(5) \times U(1)^{2}$ branch [11, 14]. Those gauge-Higgs unification can be realized in $5 \mathrm{D}$ supersymmetric models on $S^{1} / Z_{2}$ orbifold.

Interestingly, the bulk vector multiplets for such unification groups can also include the matter representations, and thus the matters can also be unified with the gauge multiplet in higher-dimensional models [13, 14. For example, the $S U(8)$ adjoint 63 includes $(\mathbf{4}, \mathbf{2}, \mathbf{1})$ and $(\overline{4}, \mathbf{1}, \mathbf{2})$ as matter representations in addition to the Higgs bidoublet $(\mathbf{1}, \mathbf{2}, \mathbf{2})$ under the $S U(4)_{c} \times S U(2)_{L} \times S U(2)_{R}$ decomposition. As a result, through the trilinear term in the bulk action (see Eq. (A.4) $)$,

$$
\int d^{2} \theta \operatorname{Tr} \frac{1}{k g^{2}}\left(-\sqrt{2} \Sigma_{1}\left[\Sigma_{2}, \Sigma_{3}\right]\right)+\text { H.C. },
$$

the Yukawa couplings for quark and lepton can be unified with the gauge couplings [15]. In the $S O(12)$ and $E_{6}$ models, the top quark Yukawa coupling can be realized in the flipped $S U(5)$ and $S U(5)$ branches, respectively [14. When $S O(16)$ bulk symmetry is considered, the second generation of the SM fermions can also be contained in the bulk gauge multiplet [13]. 


\subsection{Gauge-Higgs Unification in the NMSSM}

Next let us consider the NMSSM gauge-Higgs unification [19]. The purpose is to include the coupling $\lambda S H_{u} H_{d}$ as a zero mode interaction from the bulk action in Eq. (2.1). The $S U(3)_{W}$ adjoint includes one singlet $\mathbf{1}_{0}$ as we have seen, and the adjoint trilinear interaction $\mathbf{8}^{3}$ includes $\mathbf{2}_{1 / 2} \mathbf{2}_{-1 / 2} \mathbf{1}_{0}$. However, if the $\mathbf{1}_{0}$ component arises from the zero mode of the chiral multiplets $\Sigma_{i}$, the $\mathcal{N}=2$ supersymmetry remains in $4 \mathrm{D}$ and it is not a proper situation for our purpose. The next candidate is $S U(4)_{W}$. The $S U(4)_{W}$ symmetry is broken down to $S U(2)_{L} \times U(1)_{Y} \times U(1)^{\prime}$ and the adjoint representation is decomposed as

$$
\mathbf{1 5}=\left(\begin{array}{ccc}
\mathbf{3}_{0,0} & \mathbf{2}_{\frac{1}{2},-\frac{1}{2}} & \mathbf{2}_{\frac{1}{2}, \frac{1}{2}} \\
\mathbf{2}_{-\frac{1}{2}, \frac{1}{2}} & \mathbf{1}_{0,0} & \mathbf{1}_{0,1} \\
\mathbf{2}_{-\frac{1}{2},-\frac{1}{2}} & \mathbf{1}_{0,-1} & \mathbf{1}_{0,0}
\end{array}\right) .
$$

The two subscripts in each decomposed element stand for the hypercharges and $U(1)^{\prime}$ charges, respectively. The generators for the $U(1)_{Y}$ and $U(1)^{\prime}$ are $T_{Y}=\operatorname{diag}(1,1,-1,-1) / 4$ and $T^{\prime}=$ $\operatorname{diag}(0,0,1,-1) / 2$, respectively. In this case we can have $\lambda H_{u} H_{d} S$ interaction as part of the bulk trilinear gauge interaction. The SM singlet field $S$ has $U(1)^{\prime}$ charge. At the compactification scale we can have $g_{2}=\lambda$ where $g_{2}$ is $S U(2)_{L}$ gauge coupling. However, the weak mixing angle at the compactification scale is predicted to be $\sin ^{2} \theta_{W}=2 / 3$, which is too large to consider the RGE evolution with the MSSM particle content. Actually, the hypercharge assignment is incompatible with left-right symmetric basis as we have seen previously while the $U(1)^{\prime}$ was the proper charge assignment to obtain the left-right symmetric basis.

Employing $S U(5)_{W}$ bulk symmetry, we can obtain the NMSSM gauge-Higgs unification with proper hypercharge normalization. The $S U(5)$ adjoint representation $\mathbf{2 4}$ is decomposed under $S U(2)_{L} \times U(1)_{Y} \times U(1)_{1} \times U(1)_{2}$ gauge symmetry as

$$
\mathbf{2 4}=\left(\begin{array}{cccc}
\mathbf{3}_{Q 00} & \mathbf{2}_{Q 12} & \mathbf{2}_{Q 13} & \mathbf{2}_{Q 14} \\
\mathbf{2}_{Q 21} & \mathbf{1}_{Q 00} & \mathbf{1}_{Q 23} & \mathbf{1}_{Q 24} \\
\mathbf{2}_{Q 31} & \mathbf{1}_{Q 32} & \mathbf{1}_{Q 00} & \mathbf{1}_{Q 34} \\
\mathbf{2}_{Q 41} & \mathbf{1}_{Q 42} & \mathbf{1}_{Q 43} & \mathbf{1}_{Q 00}
\end{array}\right),
$$

where the diagonal entries, 1 triplet and 3 singlets corresponds to the unbroken generators of $S U(5)$. The subscripts $Q i j$, which are anti-symmetric $(Q i j=-Q j i)$ and $Q i i \equiv Q 00=0$, stand for the $U(1)$ charges. One can calculate $Q i j$ by the definition

$$
T \cdot \mathbf{2 4}-\mathbf{2 4} \cdot T=Q \cdot \mathbf{2 4}
$$

where $T$ is the corresponding $U(1)$ generator. We define the hypercharge generator as $T_{Y}=$ $\operatorname{diag}(1 / 2,1 / 2,0,0,-1)+\alpha(1,1,1,1,-4)$. And the adjoint trilinear coupling includes $S H_{u} H_{d}$ 


$$
\operatorname{Tr} \mathbf{2 4}^{3} \supset \mathbf{2}_{Q 12} \mathbf{1}_{Q 23} \mathbf{2}_{Q 31}
$$

We identify $\Sigma_{1}\left(\mathbf{1}_{Q 23}\right), \Sigma_{3}\left(\mathbf{2}_{Q 12}\right)$ and $\Sigma_{2}\left(\mathbf{2}_{Q 31}\right)$ as $S, H_{u}$ and $H_{d}$, respectively. The weak mixing

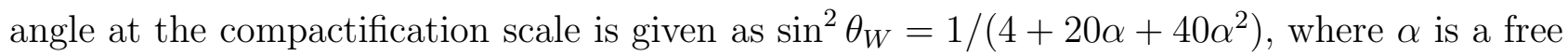
parameter in the model. The value of the weak mixing angle is not predicted in this model, but it can be consistent with its measured value.

Similar model can be constructed by using $S O(7)_{W}$, whose dimension is less then $S U(5)_{W}$. Under the decomposition $S O(7) \rightarrow S O(5) \times U(1)^{\prime}, \mathbf{2 1}=\mathbf{1 0}_{0}+\mathbf{5}_{1}+\mathbf{5}_{-1}+\mathbf{1}_{0}$, and under $S O(5) \rightarrow S U(2)_{L} \times S U(2)_{R}, \mathbf{1 0}=(\mathbf{3}, \mathbf{1})+(\mathbf{1}, \mathbf{3})+(\mathbf{2}, \mathbf{2}), \mathbf{5}=(\mathbf{2}, \mathbf{2})+(\mathbf{1}, \mathbf{1})$. Thus the adjoint trilinear term includes $S H_{u} H_{d}, \mathbf{2 1}^{3} \supset \mathbf{1 0}_{0} \mathbf{5}_{1} \mathbf{5}_{-1} \supset(\mathbf{2}, \mathbf{2})_{0}(\mathbf{2}, \mathbf{2})_{1}(\mathbf{1}, \mathbf{1})_{-1} \supset H_{u} H_{d} S$. In this case, the left-right symmetry is simply embedded, and thus the weak mixing angle can be $\sin ^{2} \theta_{W}=3 / 8$ more naturally.

One can see from Eq. (2.3) that the $S U(5)_{W}$ adjoint includes $S U(2)_{L}$ singlets and thus the adjoint trilinear term can include the singlet trilinear coupling $\kappa S S_{1} S_{2}$ as a zero mode interaction. In this case, the NMSSM superpotential,

$$
W_{\mathrm{NMSSM}}=\lambda S H_{u} H_{d}-\kappa S S_{1} S_{2}
$$

can be included in the bulk gauge interaction, and thus both $\lambda$ and $\kappa$ can be unified with gauge coupling $g_{2}$ at the compactification scale. Since the $S_{1}$ and $S_{2}$ is needed to be singlets under the SM, Q23, Q34 and $Q 42$ in Eq. (2.3) are all zero for hypercharge and thus the hypercharge generator is determined as $T_{Y}=\operatorname{diag}(3 / 2,3 / 2,-1,-1,-1) / 5$. Then the weak mixing angle is calculated as $\sin ^{2} \theta_{W}=5 / 8$, and thus extra $U(1)$ symmetry, which mixes with hypercharge, is needed to realize the proper weak mixing angle in the usual minimal unification scenario. When we consider the $S U(6)_{W}$, the NMSSM superpotential in Eq. (2.6) is included in the bulk interaction in compatible with proper weak mixing angle, since $S U(6)_{W}$ includes the trinification basis, $S U(6)_{W} \rightarrow S U(3)_{L} \times S U(3)_{R} \times U(1)^{\prime}: \mathbf{3 5}=(\mathbf{8}, \mathbf{1})+(\mathbf{1}, \mathbf{8})+(\mathbf{3}, \overline{\mathbf{3}})+(\overline{\mathbf{3}}, \mathbf{3})+$ $(\mathbf{1}, \mathbf{1})$, and $35^{3} \supset(\mathbf{1}, \mathbf{8})(\mathbf{3}, \overline{\mathbf{3}})(\overline{\mathbf{3}}, \mathbf{3})$ can include both $\lambda$ and $\kappa$ couplings. One can also use the branch $\left(F_{4}\right)_{W} \rightarrow S U(3)_{L} \times S U(3)_{R}$ to make the NMSSM superpotential. In such trinification assignment, the $B-L$ charge is also embedded in the $S U(6)_{W}$ and $\left(F_{4}\right)_{W}$, and the weak mixing angle is predicted properly. The $S O(8)_{W}$ bulk interaction which includes the NMSSM superpotential is also compatible with proper weak mixing angle, although it is not completely determined since the $B-L$ charge does not belong to $S O(8)_{W}$.

We note that there are five SM neutral complex scalar fields and three phase symmetries in the renormalizable superpotential in Eq. (2.6). One of these is related to the $U(1)_{Y}$, and the other two combinations are unwanted global symmetries, which implies that there are two 
massless Goldstone bosons after electroweak symmetry breaking. To avoid the problem, we assume that there are non-renormalizable couplings involving $S, S_{1}$ and $S_{2}$, such that the couplings break extra $U(1)$ symmetries having in the superpotential. Giving a suitable choice of charge assignment for the singlet fields, we can generate non-renormalizable couplings or

tiny mass term for $S, S_{1}$ and $S_{2}$ fields. In that way, we can make the model not having the massless Goldstone bosons in the spectrum, and the model does not have neither domain wall problem [23] nor tadpole corrections.

We also note that the renormalizable superpotential, Eq. (2.6), is different from the one in the Next to the MSSM [18]. The singlet trilinear coupling is not a self-cubic coupling contrary to the Next to the MSSM. However, as long as the orbifold model is concerned, it is more natural to have the coupling, $\kappa S S_{1} S_{2}$, if the renormalizable coupling needed in the Higgs superpotential is included in the bulk interaction. As we have seen the construction, the $\kappa$ coupling is not necessarily unified in the bulk interaction depending on the bulk gauge symmetry. If the singlet trilinear coupling is not in the bulk interaction, one can assume that the brane-localized interactions include the $S^{3}$ term having in the Next to the MSSM. In any case, it is flexible to construct such types of Non-Minimal Supersymmetric Standard Models.

In the same way as before, when the grand unified bulk gauge symmetry is considered, the Yukawa couplings for the SM fermions can also be unified with the gauge couplings in addition to the NMSSM couplings. One can consider the $S U(8)$ and $S U(9)$ bulk gauge symmetry as grand unified groups (instead of $S U(5)_{W}$ and $S U(6)_{W}$ for the electroweak sector). The $S U(8)$ bulk gauge interaction includes Eq. (2.6) with grand unification in the branch $S U(8) \rightarrow$ $S U(5)_{\text {GUT }} \times S U(3) \times U(1)^{\prime}$ (but Yukawa couplings for fermions are not unified in this case). When Pati-Salam branch of $S U(8)$ is considered, some of the Yukawa coupling for fermions and the $\lambda$ coupling in the NMSSM can be unified with the gauge couplings. In the case of $S U(9)$, the gauge couplings, Higgs trilinear couplings and the up-type or down-type Yukawa couplings for the SM fermions can be unified.

We will construct the concrete models in the next three Sections, realizing various possibilities.

\section{The $S U(8)$ Models}

To break the $S U(8)$ gauge symmetry, we choose the following $8 \times 8$ matrix representation for $R$,

$$
R=\operatorname{diag}\left(\omega^{n_{1}}, \omega^{n_{1}}, \omega^{n_{1}}, \omega^{n_{2}}, \omega^{n_{2}}, \omega^{n_{3}}, \omega^{n_{4}}, \omega^{n_{5}}\right)
$$


where $n_{i} \neq n_{j}(i \neq j)$. Then, $S U(8)$ is broken as

$$
S U(8) \rightarrow S U(3)_{c} \times S U(2)_{L} \times U(1)_{Y} \times U(1)_{1} \times U(1)_{2} \times U(1)_{3} .
$$

Without loss of generality, we can take $n_{1}=0$.

We define the generators for $U(1)_{Y} \times U(1)_{1} \times U(1)_{2} \times U(1)_{3}$ as following

$$
\begin{aligned}
T_{Y} & =\operatorname{diag}\left(-\frac{1}{3},-\frac{1}{3},-\frac{1}{3}, \frac{1}{2}, \frac{1}{2}, 0,0,0\right), \\
T_{1} & =\operatorname{diag}\left(\frac{1}{5}, \frac{1}{5}, \frac{1}{5}, \frac{1}{5}, \frac{1}{5},-\frac{1}{3},-\frac{1}{3},-\frac{1}{3}\right), \\
T_{2} & =\operatorname{diag}\left(0,0,0,0,0, \frac{1}{2},-\frac{1}{2}, 0\right), \\
T_{3} & =\operatorname{diag}\left(0,0,0,0,0, \frac{1}{2}, \frac{1}{2},-1\right) .
\end{aligned}
$$

The $S U(8)$ adjoint representation 63 is decomposed under the $S U(3)_{c} \times S U(2)_{L} \times U(1)_{Y} \times$ $U(1)_{1} \times U(1)_{2} \times U(1)_{3}$ gauge symmetry as

$$
\mathbf{6 3}=\left(\begin{array}{lllll}
(\mathbf{8}, \mathbf{1})_{Q 00} & (\mathbf{3}, \overline{\mathbf{2}})_{Q 12} & (\mathbf{3}, \mathbf{1})_{Q 13} & (\mathbf{3}, \mathbf{1})_{Q 14} & (\mathbf{3}, \mathbf{1})_{Q 15} \\
(\overline{\mathbf{3}}, \mathbf{2})_{Q 21} & (\mathbf{1}, \mathbf{3})_{Q 00} & (\mathbf{1}, \mathbf{2})_{Q 23} & (\mathbf{1}, \mathbf{2})_{Q 24} & (\mathbf{1}, \mathbf{2})_{Q 25} \\
(\overline{\mathbf{3}}, \mathbf{1})_{Q 31} & (\mathbf{1}, \overline{\mathbf{2}})_{Q 32} & (\mathbf{1}, \mathbf{1})_{Q 00} & (\mathbf{1}, \mathbf{1})_{Q 34} & (\mathbf{1}, \mathbf{1})_{Q 35} \\
(\overline{\mathbf{3}}, \mathbf{1})_{Q 41} & (\mathbf{1}, \overline{\mathbf{2}})_{Q 42} & (\mathbf{1}, \mathbf{1})_{Q 43} & (\mathbf{1}, \mathbf{1})_{Q 00} & (\mathbf{1}, \mathbf{1})_{Q 45} \\
(\overline{\mathbf{3}}, \mathbf{1})_{Q 51} & (\mathbf{1}, \overline{\mathbf{2}})_{Q 52} & (\mathbf{1}, \mathbf{1})_{Q 53} & (\mathbf{1}, \mathbf{1})_{Q 54} & (\mathbf{1}, \mathbf{1})_{Q 00}
\end{array}\right)+(\mathbf{1}, \mathbf{1})_{Q 00},
$$

where the $(\mathbf{1}, \mathbf{1})_{Q 00}$ in the third, fourth and fifth diagonal entries of the matrix, and the last term $(\mathbf{1}, \mathbf{1})_{Q 00}$ denote the gauge fields for the $U(1)_{Y} \times U(1)_{1} \times U(1)_{2} \times U(1)_{3}$ gauge symmetry. The subscripts $Q i j$, which are anti-symmetric $(Q i j=-Q j i)$, are the charges under the $U(1)_{Y} \times U(1)_{1} \times U(1)_{2} \times U(1)_{3}$ gauge symmetry, which can be easily calculated by the respective definitions of the $U(1)$ generators,

$$
\begin{aligned}
& Q 00=(0,0,0,0), \quad Q 12=\left(-\frac{5}{6}, 0,0,0\right), Q 13=\left(-\frac{1}{3}, \frac{8}{15},-\frac{1}{2},-\frac{1}{2}\right), \\
& Q 14=\left(-\frac{1}{3}, \frac{8}{15}, \frac{1}{2},-\frac{1}{2}\right), \quad Q 15=\left(-\frac{1}{3}, \frac{8}{15}, 0,1\right), \quad Q 23=\left(\frac{1}{2}, \frac{8}{15},-\frac{1}{2},-\frac{1}{2}\right), \\
& Q 24=\left(\frac{1}{2}, \frac{8}{15}, \frac{1}{2},-\frac{1}{2}\right), \quad Q 25=\left(\frac{1}{2}, \frac{8}{15}, 0,1\right), \quad Q 34=(0,0,1,0), \\
& Q 35=\left(0,0, \frac{1}{2}, \frac{3}{2}\right), \quad Q 45=\left(0,0,-\frac{1}{2}, \frac{3}{2}\right) .
\end{aligned}
$$

The $Z_{6}$ transformation property for the decomposed components of $V, \Sigma_{1}, \Sigma_{2}$, and $\Sigma_{3}$ are given in the notation in Appendix A,

$$
V^{(i j)}: \omega^{n_{i}-n_{j}}, \quad \Sigma_{1}^{(i j)}: \omega^{n_{i}-n_{j}-1}, \quad \Sigma_{2}^{(i j)}: \omega^{n_{i}-n_{j}-1-m}, \quad \Sigma_{3}^{(i j)}: \omega^{n_{i}-n_{j}+2+m} .
$$


Table 1: The zero modes of the chiral multiplets $\Sigma_{1}, \Sigma_{2}$ and $\Sigma_{3}$ in the 6 D orbifold $S U(8)$ model.

\begin{tabular}{|c|cccc|}
\hline Chiral Fields & \multicolumn{4}{|c|}{ Zero Modes } \\
\hline \hline$\Sigma_{1}$ & $Q_{X}:(\mathbf{3}, \overline{\mathbf{2}})_{Q 12} ;$ & $H_{u}^{\prime}:(\mathbf{1}, \mathbf{2})_{Q 23} ;$ & $S:(\mathbf{1}, \mathbf{1})_{Q 45} ;$ & $\bar{D}_{\delta}:(\overline{\mathbf{3}}, \mathbf{1})_{Q 51}$ \\
\hline$\Sigma_{2}$ & $D_{\delta}:(\mathbf{3}, \mathbf{1})_{Q 13} ;$ & $S_{2}:(\mathbf{1}, \mathbf{1})_{Q 34} ;$ & $\bar{D}_{X}:(\overline{\mathbf{3}}, \mathbf{1})_{Q 41} ;$ & $H_{d}:(\mathbf{1}, \overline{\mathbf{2}})_{Q 52}$ \\
\hline$\Sigma_{3}$ & $H_{u}:(\mathbf{1}, \mathbf{2})_{Q 24} ;$ & $S_{1}:(\mathbf{1}, \mathbf{1})_{Q 53} ;$ & $S_{1}^{\prime}:(\mathbf{1}, \mathbf{1})_{Q 35} ;$ & $H_{d}^{\prime}:(\mathbf{1}, \overline{\mathbf{2}})_{Q 42}$ \\
\hline
\end{tabular}

We can have several models which are quite similar. So, for simplicity, we only present one model which needs less 3-brane localized exotic quarks. These 3-brane localized exotic quarks and some extra particles from the zero modes of the chiral multiplets $\Sigma_{i}$ are vector-like under the SM gauge symmetry and can obtain the vector-like masses after the extra $U(1)_{1} \times U(1)_{2} \times U(1)_{3}$ gauge symmetry is broken at the GUT scale.

In our model, we choose $m=1$, and

$$
n_{2}=5, n_{3}=4, n_{4}=2, n_{5}=1 \text {. }
$$

The corresponding zero modes from the chiral multiplets $\Sigma_{1}, \Sigma_{2}$ and $\Sigma_{3}$ are given in the Table 11.

From the 6D bulk interaction in Eq. (2.1), we obtain the Yukawa terms

$$
\begin{gathered}
\mathcal{S}=\int d^{6} x\left[\int d ^ { 2 } \theta g _ { 6 } \left(S H_{u} H_{d}-S S_{1} S_{2}-Q_{X} \bar{D}_{X} H_{u}+S_{2} H_{u}^{\prime} H_{d}^{\prime}\right.\right. \\
\left.\left.-S_{1}^{\prime} H_{u}^{\prime} H_{d}+S_{1}^{\prime} D_{\delta} \bar{D}_{\delta}\right)+ \text { H.C. }\right]
\end{gathered}
$$

where $g_{6}$ is the $6 \mathrm{D}$ bulk gauge coupling.

Because the $U(1)_{1} \times U(1)_{2} \times U(1)_{3}$ gauge symmetry can be broken at the GUT scale, the exotic quarks $Q_{X}, \bar{D}_{X}, D_{\delta}, \bar{D}_{\delta}$, and the doublets $H_{u}^{\prime}$ and $H_{d}^{\prime}$ can become heavy after these extra $U(1)$ gauge symmetry breakings. To achieve this, on the 3 -brane at the $Z_{6}$ fixed point, for example, $z=0$, we introduce two exotic quarks $\bar{Q}_{X}^{\prime}$ and $D_{\delta}^{\prime}$ with respectively quantum numbers $(\overline{\mathbf{3}}, \mathbf{2})_{(5 / 6,0,-1,0)}$ and $(\mathbf{3}, \mathbf{1})_{(-1 / 3,8 / 15,-1,1)}$ under the $S U(3)_{C} \times S U(2)_{L} \times U(1)_{Y} \times U(1)_{1} \times$ $U(1)_{2} \times U(1)_{3}$ gauge symmetry. We also introduce a SM singlet Higgs field $\widetilde{S}_{2}$ which has the same quantum number as that of $S_{2}$ and is localized on the 3-brane at $z=0$. After $\widetilde{S}_{2}$ gets VEV, the exotic quarks and the doublets $H_{u}^{\prime}$ and $H_{d}^{\prime}$ can obtain the vector-like masses through the following 3-brane localized superpotential

$$
W=\widetilde{S}_{2} H_{u}^{\prime} H_{d}^{\prime}+\widetilde{S}_{2} D_{\delta} \bar{D}_{X}+\widetilde{S}_{2} Q_{X} \bar{Q}_{X}^{\prime}+\widetilde{S}_{2} D_{\delta}^{\prime} \bar{D}_{\delta}
$$


Similarly, $S_{1}^{\prime}$ can also become heavy, but, we require that it do not get a VEV due to the superpotential $S_{1}^{\prime} H_{u}^{\prime} H_{d}$.

Furthermore, we would like to point out that if we consider $\bar{D}_{\delta}$ as the right-handed downtype quark in the supersymmetric SM, we do not need to introduce the exotic quark $D_{\delta}^{\prime}$ on the 3-brane at $z=0$. However, $Q_{X}$ can not be considered as the quark doublet because its hypercharge is $-5 / 6$.

After the $U(1)_{1} \times U(1)_{2} \times U(1)_{3}$ gauge symmetry is broken, we can have the relevant superpotential

$$
\mathcal{S}=\int d^{6} x\left[\int d^{2} \theta g_{6}\left(S H_{u} H_{d}-S S_{1} S_{2}\right)+\text { H.C. }\right] .
$$

Integrating out the extra dimensions, we obtain the NMSSM superpotential in Eq. (2.6).

For simplicity, we assume that the compactification scale is the GUT scale and we neglect the brane-localized gauge kinetic terms which can be suppressed by large volume of the extra dimensions. The weak mixing angle is calculated to be $\sin ^{2} \theta_{W}=3 / 8$, as long as we use the definition of hypercharge generator in Eq. (3.3). The $\lambda$ and $\kappa$ couplings in the superpotential can be unified with the gauge couplings at the compactification scale,

$$
g_{1}=g_{2}=g_{3}=\lambda=\kappa=g_{6} / \sqrt{V}
$$

where $V$ is the volume of extra dimensions. The hypercharge gauge coupling is normalized as $g_{1}=\sqrt{5 / 3} g_{Y}$. However, the hypercharge normalization cannot be determined completely as long as only Higgs fields are in the bulk. In fact, $T_{Y}+\alpha T_{1}$ can be a hypercharge generator, where $\alpha$ is a free parameter. In order to determine the hypercharge normalization completely, quarks and/or leptons are needed to be from the bulk gauge multiplet. For example, if we identify $\bar{D}_{\delta}$ as the right-handed down-type quark field, $\alpha$ is determined to be zero, and the charge quantization is fixed.

We have assumed that the hypercharge generator is $S U(5)$-type unification as in Eq. (3.3). In this assignment, no Yukawa coupling for quark/lepton is included in the bulk interaction. Suppose that the hypercharge generator is redefined as

$$
T_{Y}=\operatorname{diag}\left(\frac{1}{6}, \frac{1}{6}, \frac{1}{6}, 0,0, \frac{1}{2},-\frac{1}{2},-\frac{1}{2}\right),
$$

then, the hypercharges of the zero modes in the chiral multiplets can change. We list the zero modes in chiral multiplets in Table 2 with an appropriate notation to see their hypercharges. This hypercharge generator can be identified as the Pati-Salam hypercharge assignment embedded in $S U(8)$. Then, we find that the 6D bulk interaction in Eq. (3.14) includes the top, tau and tau Dirac-neutrino Yukawa couplings,

$$
\mathcal{S}=\int d^{6} x\left[\int d ^ { 2 } \theta g _ { 6 } \left(L \bar{N} H_{u}-\bar{N} E_{\delta} \bar{E}-Q \bar{U} H_{u}+\bar{E}_{\delta} H_{d} L^{\prime}\right.\right.
$$


Table 2: The zero modes of the chiral multiplets $\Sigma_{1}, \Sigma_{2}$ and $\Sigma_{3}$ in the $6 \mathrm{D}$ orbifold $S U(8)$ model with Pati-Salam charge assignment.

\begin{tabular}{|c|cccc|}
\hline Chiral Fields & \multicolumn{4}{|c|}{ Zero Modes } \\
\hline \hline$\Sigma_{1}$ & $Q:(\mathbf{3}, \overline{\mathbf{2}})_{Q 12} ;$ & $H_{d}:(\mathbf{1}, \mathbf{2})_{Q 23} ;$ & $\bar{N}:(\mathbf{1}, \mathbf{1})_{Q 45} ;$ & $\bar{U}_{\delta}:(\overline{\mathbf{3}}, \mathbf{1})_{Q 51}$ \\
\hline$\Sigma_{2}$ & $D_{\delta}:(\mathbf{3}, \mathbf{1})_{Q 13} ;$ & $\bar{E}_{\delta}:(\mathbf{1}, \mathbf{1})_{Q 34} ;$ & $\bar{U}:(\overline{\mathbf{3}}, \mathbf{1})_{Q 41} ;$ & $L:(\mathbf{1}, \overline{\mathbf{2}})_{Q 52}$ \\
\hline$\Sigma_{3}$ & $H_{u}:(\mathbf{1}, \mathbf{2})_{Q 24} ;$ & $E_{\delta}:(\mathbf{1}, \mathbf{1})_{Q 53} ;$ & $\bar{E}:(\mathbf{1}, \mathbf{1})_{Q 35} ;$ & $L^{\prime}:(\mathbf{1}, \overline{\mathbf{2}})_{Q 42}$ \\
\hline
\end{tabular}

$$
\left.-L \bar{E} H_{d}+\bar{E} D_{\delta} \bar{U}_{\delta}\right)+ \text { H.C.] }
$$

The Dirac-neutrino Yukawa coupling can be considered as $\lambda S H_{u} H_{d}$ coupling, but the singlet trilinear term is not included since the $S_{1}$ and $S_{2}$ have non-zero hypercharges in this case. When we extend bulk gauge group, both the NMSSM superpotential and the quark/lepton Yukawa couplings can arise from the bulk gauge interaction. We will study the $S U(9)$ bulk gauge symmetry in the next two Sections.

\section{$4 \quad 6 \mathrm{D} S U(9)$ Models}

To break the $S U(9)$ gauge symmetry, we choose the following $9 \times 9$ matrix representation for $R$

$$
R=\operatorname{diag}\left(\omega^{n_{1}}, \omega^{n_{1}}, \omega^{n_{1}}, \omega^{n_{1}}, \omega^{n_{2}}, \omega^{n_{2}}, \omega^{n_{3}}, \omega^{n_{4}}, \omega^{n_{5}}\right),
$$

where $n_{i} \neq n_{j}(i \neq j)$. Without loss of generality, we choose $n_{1}=0$. Then, $S U(9)$ is broken as

$$
S U(9) \rightarrow S U(4) \times S U(2)_{L} \times U(1)^{\prime} \times U(1)_{\alpha} \times U(1)_{\beta} \times U(1)_{\gamma}
$$

We define the generators for $U(1)^{\prime} \times U(1)_{\alpha} \times U(1)_{\beta} \times U(1)_{\gamma}$ as following :

$$
\begin{aligned}
& T^{\prime}=\operatorname{diag}(0,0,0,0,0,0,+1,-1,0), \\
& T_{\alpha}=\operatorname{diag}(0,0,0,0,+1,+1,-1,-1,0), \\
& T_{\beta}=\operatorname{diag}(+1,+1,+1,+1,-1,-1,-1,-1,0), \\
& T_{\gamma}=\operatorname{diag}(+1,+1,+1,+1,+1,+1,+1,+1,-8) .
\end{aligned}
$$

The $S U(9)$ adjoint representation 80 is decomposed under the $S U(4) \times S U(2)_{L} \times U(1)^{\prime} \times$ 
$U(1)_{\alpha} \times U(1)_{\beta} \times U(1)_{\gamma}$ gauge symmetry as

$$
\mathbf{8 0}=\left(\begin{array}{ccccc}
(\mathbf{1 5}, \mathbf{1})_{Q 00} & (\mathbf{4}, \overline{\mathbf{2}})_{Q 12} & (\mathbf{4}, \mathbf{1})_{Q 13} & (\mathbf{4}, \mathbf{1})_{Q 14} & (\mathbf{4}, \mathbf{1})_{Q 15} \\
(\overline{\mathbf{4}}, \mathbf{2})_{Q 21} & (\mathbf{1}, \mathbf{3})_{Q 00} & (\mathbf{1}, \mathbf{2})_{Q 23} & (\mathbf{1}, \mathbf{2})_{Q 24} & (\mathbf{1}, \mathbf{2})_{Q 25} \\
(\overline{\mathbf{4}}, \mathbf{1})_{Q 31} & (\mathbf{1}, \overline{\mathbf{2}})_{Q 32} & (\mathbf{1}, \mathbf{1})_{Q 00} & (\mathbf{1}, \mathbf{1})_{Q 34} & (\mathbf{1}, \mathbf{1})_{Q 35} \\
(\overline{\mathbf{4}}, \mathbf{1})_{Q 41} & (\mathbf{1}, \overline{\mathbf{2}})_{Q 42} & (\mathbf{1}, \mathbf{1})_{Q 43} & (\mathbf{1}, \mathbf{1})_{Q 00} & (\mathbf{1}, \mathbf{1})_{Q 45} \\
(\overline{\mathbf{4}}, \mathbf{1})_{Q 51} & (\mathbf{1}, \overline{\mathbf{2}})_{Q 52} & (\mathbf{1}, \mathbf{1})_{Q 53} & (\mathbf{1}, \mathbf{1})_{Q 54} & (\mathbf{1}, \mathbf{1})_{Q 00}
\end{array}\right)+(\mathbf{1}, \mathbf{1})_{Q 00}
$$

where the $(\mathbf{1}, \mathbf{1})_{Q 00}$ in the third, fourth and fifth diagonal entries of the matrix, and the last term $(\mathbf{1}, \mathbf{1})_{Q 00}$ denote the gauge fields for the $U(1)^{\prime} \times U(1)_{\alpha} \times U(1)_{\beta} \times U(1)_{\gamma}$ gauge symmetry. Moreover, the subscripts $Q i j$, which are anti-symmetric $(Q i j=-Q j i)$, are the charges under the $U(1)^{\prime} \times U(1)_{\alpha} \times U(1)_{\beta} \times U(1)_{\gamma}$ gauge symmetry

$$
\begin{aligned}
& Q 00=(0,0,0,0), \quad Q 12=(0,-1,2,0), \quad Q 13=(-1,1,2,0), \\
& Q 14=(1,1,2,0), \quad Q 15=(0,0,1,9), \quad Q 23=(-1,2,0,0), \\
& Q 24=(1,2,0,0), \quad Q 25=(0,1,-1,9), \quad Q 34=(2,0,0,0), \\
& Q 35=(1,-1,-1,9), \quad Q 45=(-1,-1,-1,9) .
\end{aligned}
$$

The $Z_{6}$ transformation property for the decomposed components of $V, \Sigma_{1}, \Sigma_{2}$, and $\Sigma_{3}$ are given similarly as those in Eq. (3.12).

We can have several models which are quite similar. So, for simplicity, we only present one model with the up-type partial Yukawa unification, and one similar model with the down-type partial Yukawa unification. There are some extra particles from the zero modes of the chiral multiplets $\Sigma_{i}$. To give them very heavy masses, we introduce 3-brane localized additional particles. These 3-brane localized additional particles and the extra particles from the zero modes of the chiral multiplets $\Sigma_{i}$ are vector-like under the SM gauge symmetry and can obtain the vector-like masses after the $U(1)^{\prime}$ gauge symmetry is broken at the GUT scale.

\subsection{Up-Type Partial Unification}

We define the generator for $U(1)_{I_{3 R}}$ as

$$
\begin{aligned}
T_{I_{3 R}} & \equiv \frac{1}{4} T_{\alpha}+\frac{1}{8} T_{\beta}+\frac{1}{24} T_{\gamma} \\
& =\operatorname{diag}\left(+\frac{1}{6},+\frac{1}{6},+\frac{1}{6},+\frac{1}{6},+\frac{1}{6},+\frac{1}{6},-\frac{1}{3},-\frac{1}{3},-\frac{1}{3}\right) .
\end{aligned}
$$

Then, $\operatorname{Tr}\left[T_{I_{3 R}}^{2}\right]=1 / 2$.

We assume that the $S U(4) \times S U(2)_{L} \times U(1)_{I_{3 R}}$ gauge symmetry is broken down to the SM gauge symmetry at the compactification scale by the Higgs fields with quantum numbers 
Table 3: The zero modes of the chiral multiplets $\Sigma_{1}, \Sigma_{2}$ and $\Sigma_{3}$ in the $6 \mathrm{D}$ orbifold $S U(9)$ model with up-type partial unification.

\begin{tabular}{|c|cccc|}
\hline Chiral Fields & \multicolumn{4}{|c|}{ Zero Modes } \\
\hline \hline$\Sigma_{1}$ & $L_{3}:(\mathbf{4}, \overline{\mathbf{2}})_{Q 12} ;$ & $H_{u}^{\prime}:(\mathbf{1}, \mathbf{2})_{Q 24} ;$ & $S_{1}^{\prime}:(\mathbf{1}, \mathbf{1})_{Q 35} ;$ & $S:(\mathbf{1}, \mathbf{1})_{Q 43}$ \\
\hline$\Sigma_{2}$ & $L_{2}:(\mathbf{4}, \overline{\mathbf{2}})_{Q 12} ;$ & $H_{u}:(\mathbf{1}, \mathbf{2})_{Q 24} ;$ & $S_{1}:(\mathbf{1}, \mathbf{1})_{Q 35} ;$ & $S^{\prime}:(\mathbf{1}, \mathbf{1})_{Q 43}$ \\
\hline$\Sigma_{3}$ & $L_{X}:(\mathbf{4}, \mathbf{1})_{Q 15} ;$ & $H_{d}:(\mathbf{1}, \overline{\mathbf{2}})_{Q 32} ;$ & $R_{3}^{u}:(\overline{\mathbf{4}}, \mathbf{1})_{Q 41} ;$ & $S_{2}:(\mathbf{1}, \mathbf{1})_{Q 54}$ \\
\hline
\end{tabular}

$(\overline{\mathbf{4}}, \mathbf{1},-1 / 2)$ and $(\mathbf{4}, \mathbf{1}, 1 / 2)$ under the $S U(4) \times S U(2)_{L} \times U(1)_{I_{3 R}}$ gauge symmetry, i.e., the same quantum numbers as those of the right-handed neutrino and its Hermitian conjugate. Then, we obtain that $\sin ^{2} \theta_{W}=3 / 8$ at the unification scale. The $U(1)_{B-L}$ generator is embedded in $S U(4)$ as

$$
T_{B-L}=\operatorname{diag}\left(\frac{1}{3}, \frac{1}{3}, \frac{1}{3},-1,0,0,0,0,0\right) .
$$

The hypercharge generator is $T_{Y}=T_{I_{3 R}}+\frac{1}{2} T_{B-L}$, and the normalization is determined as $g_{Y}=\sqrt{3 / 5} g_{1}$.

For the transformation, Eq. (3.12) of the vector and the chiral multiplets, we choose $m=0$ and

$$
n_{2}=5, \quad n_{3}=3, \quad n_{4}=4, \quad n_{5}=2 .
$$

The zero modes from the chiral multiplets $\Sigma_{1}, \Sigma_{2}$ and $\Sigma_{3}$ are given in the Table 3, It is easy to check that for $L_{2}, L_{3}, S, S^{\prime}, S_{1}, S_{1}^{\prime}$ and $S_{2}$, the $U(1)_{I_{3 R}}$ charges are zero; for $H_{u}, H_{u}^{\prime}$ and $L_{X}$, the $U(1)_{I_{3 R}}$ charges are $1 / 2$; and for $H_{d}$ and $R_{3}^{u}$, the $U(1)_{I_{3 R}}$ charges are $-1 / 2$.

From the trilinear term in the $6 \mathrm{D}$ bulk action in Eq. (2.1), we obtain the Yukawa terms

$$
\begin{gathered}
\mathcal{S}=\int d^{6} x\left[\int d ^ { 2 } \theta g _ { 6 } \left(L_{3} R_{3}^{u} H_{u}+L_{2} R_{3}^{u} H_{u}^{\prime}+S H_{d} H_{u}+S^{\prime} H_{d} H_{u}^{\prime}\right.\right. \\
\left.\left.+S S_{1} S_{2}+S^{\prime} S_{1}^{\prime} S_{2}\right)+ \text { H.C. }\right]
\end{gathered}
$$

The $L_{2}$ can be considered as the left-handed fermions in the second family in the SM. Because the $U(1)^{\prime}$ gauge symmetry can be broken at the GUT scale, the extra particles $H_{u}^{\prime}$, $L_{X}, S^{\prime}$ and $S_{1}^{\prime}$ can become very heavy after it is broken. In addition, although we can not distinguish the fields $\left(H_{u}, H_{u}^{\prime}\right),\left(S, S^{\prime}\right)$ and $\left(S_{1}, S_{1}^{\prime}\right)$ by gauge symmetry, we can distinguish them via the $R$ symmetry [24]. The $R$ symmetry for the $T^{2} / Z_{6}$ orbifold is $S O(2)_{56} \times U(1)_{4_{+}} \times$ $U(1)_{4_{-}}$. Under this $R$ symmetry, the quantum numbers for $z, \theta, V, \Sigma_{1}, \Sigma_{2}$, and $\Sigma_{3}$ are $(1,0,0),(-1 / 2,-1 / 2,0),(0,0,0),(-1,0,0),(0,-1 / 2,-1 / 2)$, and $(0,-1 / 2,1 / 2)$, respectively (For details, see Ref. [24].). To give the masses to $H_{u}^{\prime}$ and $L_{X}$, on the 3 -brane at the $Z_{6}$ fixed 
Table 4: The zero modes of the chiral multiplets $\Sigma_{1}, \Sigma_{2}$ and $\Sigma_{3}$ in the $6 \mathrm{D}$ orbifold $S U(9)$ model with down-type partial unification.

\begin{tabular}{|c|cccc|}
\hline Chiral Fields & \multicolumn{4}{|c|}{ Zero Modes } \\
\hline \hline$\Sigma_{1}$ & $L_{3}:(\mathbf{4}, \overline{\mathbf{2}})_{Q 12} ;$ & $H_{d}^{\prime}:(\mathbf{1}, \mathbf{2})_{Q 24} ;$ & $S_{1}^{\prime}:(\mathbf{1}, \mathbf{1})_{Q 35} ;$ & $S:(\mathbf{1}, \mathbf{1})_{Q 43}$ \\
\hline$\Sigma_{2}$ & $L_{2}:(\mathbf{4}, \overline{\mathbf{2}})_{Q 12} ;$ & $H_{d}:(\mathbf{1}, \mathbf{2})_{Q 24} ;$ & $S_{1}:(\mathbf{1}, \mathbf{1})_{Q 35} ;$ & $S^{\prime}:(\mathbf{1}, \mathbf{1})_{Q 43}$ \\
\hline$\Sigma_{3}$ & $L_{X}:(\mathbf{4}, \mathbf{1})_{Q 15} ;$ & $H_{u}:(\mathbf{1}, \overline{\mathbf{2}})_{Q 32} ;$ & $R_{3}^{d}:(\overline{\mathbf{4}}, \mathbf{1})_{Q 41} ;$ & $S_{2}:(\mathbf{1}, \mathbf{1})_{Q 54}$ \\
\hline
\end{tabular}

point, for example, $z=0$, we introduce three 3 -brane localized fields $\widetilde{S}, \widetilde{H}_{d}$ and $\widetilde{R}_{X}$ with respectively gauge quantum numbers $(\mathbf{1}, \mathbf{1})_{(-1,0,0,0)},(\mathbf{1}, \mathbf{2})_{(0,-2,0,0)}$ and $(\overline{\mathbf{4}}, \mathbf{1})_{(1,0,-1,-9)}$ under the $S U(4) \times S U(2)_{L} \times U(1)^{\prime} \times U(1)_{\alpha} \times U(1)_{\beta} \times U(1)_{\gamma}$ gauge symmetry. We also assume that under the $R$ symmetry, the quantum numbers for $\widetilde{S}, \widetilde{H}_{d}$ and $\widetilde{R}_{X}$ are $(0,-1 / 2,-1 / 2),(0,-1 / 2,1 / 2)$, and $(-1,0,0)$, respectively. After $\widetilde{S}$ gets VEV, the $\left(H_{u}^{\prime}, \widetilde{H}_{d}\right)$ and $\left(L_{X}, \widetilde{R}_{X}\right)$ can obtain the vector-like masses through the following 3-brane localized superpotential

$$
W=\widetilde{S} H_{u}^{\prime} \widetilde{H}_{d}+\widetilde{S} L_{X} \widetilde{R}_{X}
$$

However, the term $\widetilde{S} H_{u} \widetilde{H}_{d}$ is forbidden by the $R$ symmetry. Similarly, $S^{\prime}$ and $S_{1}^{\prime}$ can also become very heavy.

In short, after the $U(1)^{\prime}$ gauge symmetry is broken, we can have the relevant superpotential

$$
\mathcal{S}=\int d^{6} x\left[\int d^{2} \theta g_{6}\left(L_{3} R_{3}^{u} H_{u}+S H_{d} H_{u}+S S_{1} S_{2}\right)+\text { H.C. }\right] .
$$

giving rise to the unification of the gauge couplings with $\lambda, \kappa$ and $y_{t}$.

\subsection{Down-Type Partial Unification}

We define the generator for $U(1)_{I_{3 R}}$ as

$$
\begin{aligned}
T_{I_{3 R}} & \equiv-\frac{1}{4} T_{\alpha}-\frac{1}{8} T_{\beta}-\frac{1}{24} T_{\gamma} \\
& =\operatorname{diag}\left(-\frac{1}{6},-\frac{1}{6},-\frac{1}{6},-\frac{1}{6},-\frac{1}{6},-\frac{1}{6},+\frac{1}{3},+\frac{1}{3},+\frac{1}{3}\right) .
\end{aligned}
$$

Similar to the above up-type partial unification model, we choose $m=0$ and $n_{i}$ in Eq. (4.14). The zero modes from the chiral multiplets $\Sigma_{1}, \Sigma_{2}$ and $\Sigma_{3}$ are given in the Table 4 . It is easy to check that for $L_{2}, L_{3}, S, S^{\prime}, S_{1}, S_{1}^{\prime}$ and $S_{2}$, the $U(1)_{I_{3 R}}$ charges are zero; for $H_{u}$ and $R_{3}^{d}$, the $U(1)_{I_{3 R}}$ charges are $1 / 2$; and for $H_{d}, H_{d}^{\prime}$ and $L_{X}$, the $U(1)_{I_{3 R}}$ charges are $-1 / 2$. 
From the trilinear terms in the $6 \mathrm{D}$ bulk action, we obtain the Yukawa terms

$$
\begin{gathered}
\mathcal{S}=\int d^{6} x\left[\int d ^ { 2 } \theta g _ { 6 } \left(L_{3} R_{3}^{d} H_{d}+L_{2} R_{3}^{d} H_{d}^{\prime}+S H_{d} H_{u}+S^{\prime} H_{d}^{\prime} H_{u}\right.\right. \\
\left.\left.+S S_{1} S_{2}+S^{\prime} S_{1}^{\prime} S_{2}\right)+ \text { H.C. }\right]
\end{gathered}
$$

Similar to the above up-type partial unification model, $L_{2}$ can be considered as the lefthanded fermions in the second family in the SM, and the extra particles $H_{d}^{\prime}, L_{X}, S^{\prime}$ and $S_{1}^{\prime}$ can become very heavy after the $U(1)^{\prime}$ gauge symmetry is broken at the GUT scale. Thus, after the $U(1)^{\prime}$ gauge symmetry is broken, we can have the relevant superpotential

$$
\mathcal{S}=\int d^{6} x\left[\int d^{2} \theta g_{6}\left(L_{3} R_{3}^{d} H_{d}+S H_{d} H_{u}+S S_{1} S_{2}\right)+\text { H.C. }\right] .
$$

giving rise to the unification of the gauge couplings with $\lambda, \kappa$ and $y_{b}$.

As a remark, to break the $S U(4) \times S U(2)_{L} \times U(1)_{I_{3 R}}$ gauge symmetry down to the SM gauge symmetry by Higgs mechanism in our models, on the 3-brane at $z=0$, we emphasize that we introduce the Higgs fields with the same quantum numbers as those of the right-handed neutrino and its Hermitian conjugate. Of course, if we did not specify the quantum numbers of the Higgs fields, we can not distinguish the up-type partial unification and down-type partial unification models.

\section{$5 \quad$ 7D $S U(9)$ Models}

In $6 \mathrm{D} S U(9)$ models on $T^{2} / Z_{6}$ orbifold in previous Section, unwanted fields remain in the zero mode spectrum after the orbifold projection. When the models are constructed in 7D, the orbifold projection is more powerful, and the unwanted fields can be projected out.

We consider the $7 \mathrm{D}$ space-time $M^{4} \times T^{2} / Z_{6} \times S^{1} / Z_{2}$. The coordinates are $x^{\mu}(\mu=0,1,2,3)$, $x^{5}, x^{6}$ and $x^{7}$. Because $T^{2}$ is homeomorphic to $S^{1} \times S^{1}$, we assume that the radii for the circles along the $x^{5}, x^{6}$ and $x^{7}$ directions are $R_{1}, R_{2}$, and $R^{\prime}$, respectively. We define the complex coordinate $z$ for $T^{2}$ and the real coordinate $y$ for $S^{1}$

$$
z \equiv \frac{1}{2}\left(x^{5}+i x^{6}\right), \quad y \equiv x^{7} .
$$

The $T^{2} / Z_{6}$ orbifold is defined in the Appendix A, and the $S^{1} / Z_{2}$ orbifold is obtained from $S^{1}$ by moduloing the equivalent class

$$
\Gamma_{S}: \quad y \sim-y
$$

There are two fixed points: $y=0$ and $y=\pi R^{\prime}$. 
The $\mathcal{N}=1$ supersymmetry in 7D has 16 supercharges and corresponds to the $\mathcal{N}=4$ supersymmetry in $4 \mathrm{D}$, thus, only the gauge multiplet can be introduced in the bulk. This multiplet can be decomposed under the $4 \mathrm{D} \mathcal{N}=1$ supersymmetry into a vector multiplet $V$ and three chiral multiplets $\Sigma_{1}, \Sigma_{2}$, and $\Sigma_{3}$ in the adjoint representation, where the fifth and sixth components of the gauge field, $A_{5}$ and $A_{6}$ are contained in the lowest component of $\Sigma_{1}$, and the seventh component of the gauge field $A_{7}$ is contained in the lowest component of $\Sigma_{2}$.

For the bulk gauge group $G$, we write down the bulk action in the Wess-Zumino gauge and $4 \mathrm{D} \mathcal{N}=1$ supersymmetry language [25]

$$
\begin{aligned}
\mathcal{S}= & \int d^{7} x\left\{\operatorname { T r } \left[\int d^{2} \theta\left(\frac{1}{4 k g^{2}} \mathcal{W}^{\alpha} \mathcal{W}_{\alpha}+\frac{1}{k g^{2}}\left(\Sigma_{3} \partial_{z} \Sigma_{2}+\Sigma_{1} \partial_{y} \Sigma_{3}-\sqrt{2} \Sigma_{1}\left[\Sigma_{2}, \Sigma_{3}\right]\right)\right)\right.\right. \\
& + \text { H.C. }]+\int d^{4} \theta \frac{1}{k g^{2}} \operatorname{Tr}\left[\left(\frac{1}{\sqrt{2}} \partial_{z}^{\dagger}+\Sigma_{1}^{\dagger}\right) e^{-2 V}\left(-\frac{1}{\sqrt{2}} \partial_{z}+\Sigma_{1}\right) e^{2 V}+\frac{1}{4} \partial_{z}^{\dagger} e^{-2 V} \partial_{z} e^{2 V}\right. \\
& \left.\left.+\left(\frac{1}{\sqrt{2}} \partial_{y}+\Sigma_{2}^{\dagger}\right) e^{-2 V}\left(-\frac{1}{\sqrt{2}} \partial_{y}+\Sigma_{2}\right) e^{2 V}+\frac{1}{4} \partial_{y} e^{-2 V} \partial_{y} e^{2 V}+\Sigma_{3}^{\dagger} e^{-2 V} \Sigma_{3} e^{2 V}\right]\right\}
\end{aligned}
$$

From above action, we obtain the transformations of vector multiplet

$$
\begin{aligned}
V\left(x^{\mu}, \omega z, \omega^{-1} \bar{z}, y\right) & =R V\left(x^{\mu}, z, \bar{z}, y\right) R^{-1}, \\
\Sigma_{1}\left(x^{\mu}, \omega z, \omega^{-1} \bar{z}, y\right) & =\omega^{-1} R \Sigma_{1}\left(x^{\mu}, z, \bar{z}, y\right) R^{-1}, \\
\Sigma_{2}\left(x^{\mu}, \omega z, \omega^{-1} \bar{z}, y\right) & =R \Sigma_{2}\left(x^{\mu}, z, \bar{z}, y\right) R^{-1}, \\
\Sigma_{3}\left(x^{\mu}, \omega z, \omega^{-1} \bar{z}, y\right) & =\omega R \Sigma_{3}\left(x^{\mu}, z, \bar{z}, y\right) R^{-1}, \\
V\left(x^{\mu}, z, \bar{z},-y\right) & =P V\left(x^{\mu}, z, \bar{z}, y\right) P^{-1}, \\
\Sigma_{1}\left(x^{\mu}, z, \bar{z},-y\right) & =P \Sigma_{1}\left(x^{\mu}, z, \bar{z}, y\right) P^{-1}, \\
\Sigma_{2}\left(x^{\mu}, z, \bar{z},-y\right) & =-P \Sigma_{2}\left(x^{\mu}, z, \bar{z}, y\right) P^{-1}, \\
\Sigma_{3}\left(x^{\mu}, z, \bar{z},-y\right) & =-P \Sigma_{3}\left(x^{\mu}, z, \bar{z}, y\right) P^{-1},
\end{aligned}
$$

where we introduce the non-trivial $R$ and $P$ to break the bulk gauge group.

To break the $S U(9)$ gauge symmetry, we choose the following $9 \times 9$ matrix representations for $R$ and $P$

$$
\begin{aligned}
& R=\operatorname{diag}\left(+1,+1,+1,+1, \omega^{n_{1}}, \omega^{n_{1}}, \omega^{n_{1}}, \omega^{n_{2}}, \omega^{n_{2}}\right), \\
& P=\operatorname{diag}(+1,+1,+1,+1,+1,+1,-1,-1,+1),
\end{aligned}
$$

where $n_{1} \neq n_{2} \neq 0$. Then, we obtain

$$
\begin{aligned}
S U(9) / R & =S U(4) \times S U(3) \times S U(2) \times U(1)^{2}, \\
S U(9) / P & =S U(7) \times S U(2) \times U(1), \\
S U(9) /\{R \cup P\} & =S U(4) \times S U(2)_{L} \times U(1)^{\prime} \times U(1)_{\alpha} \times U(1)_{\beta} \times U(1)_{\gamma},
\end{aligned}
$$


Table 5: The zero modes of the chiral multiplets $\Sigma_{1}, \Sigma_{2}$ and $\Sigma_{3}$ in the $7 \mathrm{D}$ orbifold $S U(9)$ model with up-type partial unification.

\begin{tabular}{|c|cccc|}
\hline Chiral Fields & \multicolumn{4}{|c|}{ Zero Modes } \\
\hline \hline$\Sigma_{1}$ & $L_{3}:(\mathbf{4}, \overline{\mathbf{2}})_{Q 12} ;$ & $X_{1}:(\mathbf{1}, \mathbf{2})_{Q 25} ;$ & $S:(\mathbf{1}, \mathbf{1})_{Q 34}$ \\
\hline$\Sigma_{2}$ & $H_{u}:(\mathbf{1}, \mathbf{2})_{Q 23} ;$ & $X_{2}:(\mathbf{1}, \overline{\mathbf{2}})_{Q 32} ;$ & $S_{1}:(\mathbf{1}, \mathbf{1})_{Q 45} ;$ & $S_{X}:(\mathbf{1}, \mathbf{1})_{Q 54}$ \\
\hline$\Sigma_{3}$ & $R_{3}^{u}:(\overline{\mathbf{4}}, \mathbf{1})_{Q 31} ;$ & $H_{d}:(\mathbf{1}, \overline{\mathbf{2}})_{Q 42} ;$ & $S_{2}:(\mathbf{1}, \mathbf{1})_{Q 53}$ \\
\hline
\end{tabular}

where the quotient $G / H$ denotes the commutant of $H$ in $G$. The generators for the $U(1)^{\prime} \times$ $U(1)_{\alpha} \times U(1)_{\beta} \times U(1)_{\gamma}$ gauge symmetry are defined in Eqs. (4.4)-(4.6).

The $Z_{6} \times Z_{2}$ transformation property for the decomposed components of $V$ is

$$
V:\left(\begin{array}{ccccc}
(1,+) & \left(\omega^{-n_{1}},+\right) & \left(\omega^{-n_{1}},-\right) & \left(\omega^{-n_{2}},-\right) & \left(\omega^{-n_{2}},+\right) \\
\left(\omega^{n_{1}},+\right) & (1,+) & (1,-) & \left(\omega^{n_{1}-n_{2}},-\right) & \left(\omega^{n_{1}-n_{2}},+\right) \\
\left(\omega^{n_{1}},-\right) & (1,-) & (1,+) & \left(\omega^{n_{1}-n_{2}},+\right) & \left(\omega^{n_{1}-n_{2}},-\right) \\
\left(\omega^{n_{2}},-\right) & \left(\omega^{n_{2}-n_{1}},-\right) & \left(\omega^{n_{2}-n_{1}},+\right) & (1,+) & (1,-) \\
\left(\omega^{n_{2}},+\right) & \left(\omega^{n_{2}-n_{1}},+\right) & \left(\omega^{n_{2}-n_{1}},-\right) & (1,-) & (1,+)
\end{array}\right)+(1,+)
$$

and the transformation properties for the chiral multiplets, $\Sigma_{i}$, are also obtained from Eqs. (5.5)(5.11). The zero modes transform as $(1,+)$.

Similar to the $6 \mathrm{D}$ up-type partial unification model, the generator for the $U(1)_{I_{3 R}}$ gauge symmetry is defined in Eq. (4.12).

We choose $n_{1}=5$ and $n_{2}=4$. The zero modes from the chiral multiplets $\Sigma_{1}, \Sigma_{2}$ and $\Sigma_{3}$ are given in the Table 5. It is easy to check that for $L_{3}, S, S_{1}, S_{2}$ and $S_{X}$, the $U(1)_{I_{3 R}}$ charges are zero; for $H_{u}$ and $X_{1}$, the $U(1)_{I_{3 R}}$ charges are $1 / 2$; and for $H_{d}, R_{3}^{u}$ and $X_{2}$, the $U(1)_{I_{3 R}}$ charges are $-1 / 2$.

From the following trilinear term in the $7 \mathrm{D}$ bulk action

$$
\mathcal{S}=\int d^{7} x\left[\int d^{2} \theta \frac{1}{k} \operatorname{Tr}\left(-\sqrt{2} g \Sigma_{1}\left[\Sigma_{2}, \Sigma_{3}\right]\right)+\text { H.C. }\right]
$$

we obtain the Yukawa terms

$$
\mathcal{S}=\int d^{7} x\left[\int d^{2} \theta g_{7}\left(L_{3} R_{3}^{u} H_{u}+S H_{d} H_{u}+S S_{1} S_{2}+S_{2} X_{1} X_{2}\right)+\text { H.C. }\right] .
$$

Because the $U(1)^{\prime}$ gauge symmetry can be broken at the GUT scale, the extra particles $X_{1}, X_{2}$ and $S_{X}$ can become very heavy after it is broken. For simplicity, we do not consider the $R$ symmetry here. To give the masses to $X_{1}$ and $X_{2}$, on the 3-brane at $z=0$ and $y=0$, we introduce three 3 -brane localized fields $\widetilde{S}, \widetilde{X}_{1}$ and $\widetilde{X}_{2}$ with respectively quantum numbers 
$(\mathbf{1}, \mathbf{1})_{(-1,0,0,0)},(\mathbf{1}, \mathbf{2})_{(1,-1,1,-9)}$ and $(\mathbf{1}, \mathbf{2})_{(0,2,0,0)}$ under the $S U(4) \times S U(2)_{L} \times U(1)^{\prime} \times U(1)_{\alpha} \times$ $U(1)_{\beta} \times U(1)_{\gamma}$ gauge symmetry. After $\widetilde{S}$ gets VEV, the fields $\left(X_{1}, \widetilde{X}_{1}\right)$ and $\left(X_{2}, \widetilde{X}_{2}\right)$ can obtain the vector-like masses through the following 3 -brane localized superpotential

$$
W=\widetilde{S} X_{1} \widetilde{X}_{1}+\widetilde{S} X_{2} \widetilde{X}_{2}
$$

Similarly, $S_{X}$ can also become very heavy.

In short, after the $U(1)^{\prime}$ gauge symmetry is broken, we can have the relevant superpotential

$$
\mathcal{S}=\int d^{7} x\left[\int d^{2} \theta g_{7}\left(L_{3} R_{3}^{u} H_{u}+S H_{d} H_{u}+S S_{1} S_{2}\right)+\text { H.C. }\right] .
$$

Similar to the previous Section, the down-type partial unification can be obtained just by flipping the sign of $T_{I_{3 R}}$.

\section{$6 \quad$ Numerical Studies for Coupling Unifications}

\subsection{Predictions for the NMSSM Couplings}

As was pointed out, below the compactification scale we can have only the NMSSM-like particle content in Higgs sector: $H_{u}$ and $H_{d}$ the SM Higgs doublets and $S$ the SM singlet. The coupling $\lambda$ for $S H_{u} H_{d}$ is unified with the gauge couplings at the compactification scale. When the bulk gauge symmetry is extended, extra SM singlets, $S_{1}$ and $S_{2}$, can also be included in the massless modes below the compactification scale and form a singlet trilinear coupling. Therefore, when a bulk gauge symmetry with an enough large rank is considered, the NMSSM superpotential,

$$
W_{\mathrm{NMSSM}}=\lambda H_{u} H_{d} S-\kappa S S_{1} S_{2},
$$

is naturally generated from the bulk gauge interaction. Assuming that the compactification scale and gauge coupling unification scale $\left(M_{G U T}\right)$ are the same in the unified models, we have the following condition,

$$
g_{1}=g_{2}=g_{3}=\lambda=\kappa .
$$

Due to a crucial reduction of the number of the fundamental parameters from the gaugetrilinear Higgs coupling unification, we are lead immediately to a series of the very distinctive predictions (in absence of any large supersymmetric threshold corrections). Using the values of the electroweak parameters $\sin ^{2} \theta_{W}=0.23120 \pm 0.00015$ and $1 / \alpha_{E M}=127.918 \pm 0.018$ in $\overline{M S}$ scheme at $M_{Z}$ scale [26, we can determine the unification scale and the unified coupling constant. Then, evolving the remaining couplings from the unification scale to the low energy, we predict $\kappa$ and $\lambda$ as functions of $\tan \beta$ (see Fig. 䧃) where $\tan \beta \equiv\left\langle H_{u}^{0}\right\rangle /\left\langle H_{d}^{0}\right\rangle$. In our numerical 
calculations, we have used the one-loop RGEs for $\kappa$ and $\lambda$ and two-loop RGEs for Yukawa and gauge couplings 27 ]

$$
\begin{aligned}
\frac{d \alpha_{\lambda}}{d t} & =\frac{\alpha_{\lambda}}{2 \pi}\left(\alpha_{\kappa}+4 \alpha_{\lambda}+3 \alpha_{t}+3 \alpha_{b}+\alpha_{\tau}-\frac{3}{5} \alpha_{1}-3 \alpha_{2}\right) \\
\frac{d \alpha_{\kappa}}{d t} & =\frac{\alpha_{\kappa}}{2 \pi}\left(3 \alpha_{\kappa}+2 \alpha_{\lambda}\right) \\
\frac{d \alpha_{t}}{d t} & =\left[\frac{d \alpha_{t}}{d t}\right]_{\mathrm{MSSM}}+\frac{\alpha_{t}}{2 \pi}\left(\alpha_{\lambda}-\frac{1}{4 \pi} \alpha_{\lambda}\left(3 \alpha_{t}+4 \alpha_{b}+\alpha_{\tau}+3 \alpha_{\lambda}+\alpha_{\kappa}\right)\right) \\
\frac{d \alpha_{b}}{d t} & =\left[\frac{d \alpha_{b}}{d t}\right]_{\mathrm{MSSM}}+\frac{\alpha_{b}}{2 \pi}\left(\alpha_{\lambda}-\frac{1}{4 \pi} \alpha_{\lambda}\left(4 \alpha_{t}+3 \alpha_{b}+3 \alpha_{\lambda}+\alpha_{\kappa}\right)\right) \\
\frac{d \alpha_{\tau}}{d t} & =\left[\frac{d \alpha_{\tau}}{d t}\right]_{\mathrm{MSSM}}+\frac{\alpha_{\tau}}{2 \pi}\left(\alpha_{\lambda}-\frac{1}{4 \pi} \alpha_{\lambda}\left(3 \alpha_{t}+3 \alpha_{\tau}+3 \alpha_{\lambda}+\alpha_{\kappa}\right)\right) \\
\frac{d \alpha_{2}}{d t} & =\left[\frac{d \alpha_{2}}{d t}\right]_{\mathrm{MSSM}}+\frac{\alpha_{2}^{2}}{8 \pi^{2}}\left(-2 \alpha_{\lambda}\right) \\
\frac{d \alpha_{1}}{d t} & =\left[\frac{d \alpha_{1}}{d t}\right]_{\mathrm{MSSM}}+\frac{\alpha_{1}^{2}}{8 \pi^{2}}\left(-\frac{6}{5} \alpha_{\lambda}\right)
\end{aligned}
$$

where $t$ is the $\log$ of renormalization scale, $\alpha_{i}=g_{i}^{2} /(4 \pi), \alpha_{t, b, \tau}=y_{t, b, \tau}^{2} /(4 \pi), \alpha_{\lambda}=\lambda^{2} /(4 \pi)$, $\alpha_{\kappa}=\kappa^{2} /(4 \pi)$, and bracket [] MSSM denotes the corresponding two-loop RGEs in the MSSM. The $\lambda$ coupling depends on $\tan \beta$, while the $\kappa$ coupling less depends on $\tan \beta$ and is predicted to be from 0.5 to 0.55 . For large $\tan \beta$, the $\lambda$ coupling at low energy is sensitive to the running bottom-tau Yukawa couplings. In Fig. 1, we neglect the possible large supersymmetry threshold corrections to bottom quark mass.

Since the value of $\lambda$ constant at low energy scale is predicted, we can calculate upper bound on the lightest CP-even neutral Higgs mass. We use the following analytic formula which includes full one-loop and the dominant two-loop top/stop corrections [28]:

$$
\begin{aligned}
m_{h}^{2} \simeq & M_{Z}^{2}\left(\cos ^{2} 2 \beta+\frac{2 \lambda^{2}}{g^{\prime 2}+g_{2}^{2}} \sin ^{2} 2 \beta\right)\left(1-\frac{3 \bar{m}_{t}^{2}}{8 \pi^{2} v^{2}} t\right) \\
& +\frac{3 \bar{m}_{t}^{4}}{4 \pi^{2} v^{2}}\left(\frac{1}{2} X_{t}+t+\frac{1}{16 \pi^{2}}\left(\frac{3}{2} \frac{\bar{m}_{t}^{2}}{v^{2}}-32 \pi \alpha_{3}\right)\left(X_{t}+t\right) t\right)
\end{aligned}
$$

where $t=\log \left(M_{S}^{2} / m_{t}^{2}\right), v=174 \mathrm{GeV}$ is the usual SM Higgs VEV, and

$$
X_{t}=\frac{2 \tilde{A}_{t}^{2}}{M_{S}^{2}}\left(1-\frac{\tilde{A}_{t}^{2}}{12 M_{S}^{2}}\right),
$$

where $\tilde{A}_{t}=A_{t}-\mu \cot \beta$ is the top squark mixing parameter and $\mu$ is the supersymmetric Higgs mass parameter. The supersymmetric scale, $M_{S}^{2}=\left(M_{\tilde{t}_{1}}^{2}+M_{\tilde{t}_{2}}^{2}\right) / 2$, is the average of the two stop squared-masses. The $\bar{m}_{t}$ is the running top quark mass at $\bar{m}_{t}$ in $\overline{M S}$ scheme. In 


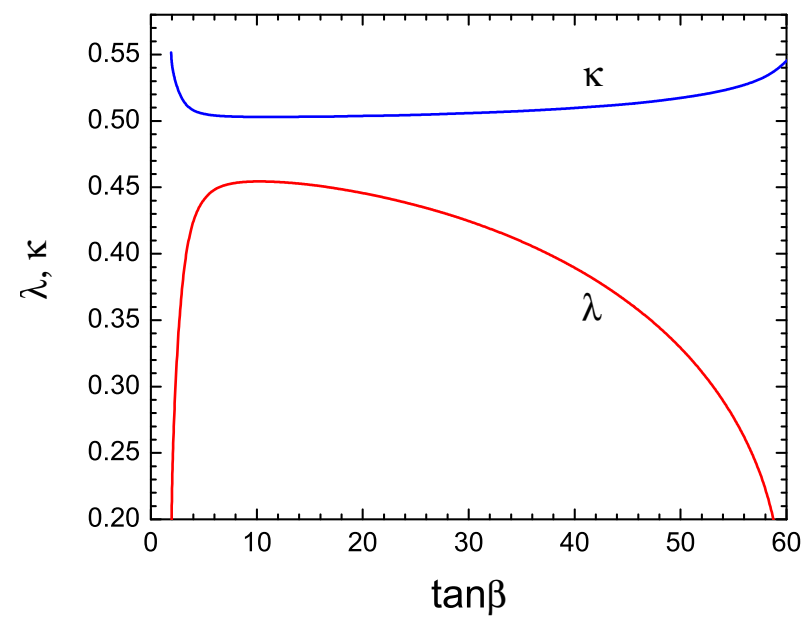

Figure 1: The Higgs trilinear couplings $\lambda$ and $\kappa$ (red and blue lines respectively) versus the $\tan \beta$. We use top quark mass to be $178 \mathrm{GeV}$.

our calculations, we use the top quark pole mass $m_{t}=178 \mathrm{GeV}$, and $M_{S}=1 \mathrm{TeV}$. From the Eq. (6.11), we can see that the maximal value for $X_{t}$ is $X_{t}=6$, and we use this value to calculate the lightest CP-even Higgs mass upper bounds in various models presented in Fig. 2 ,

\subsection{Predictions for Yukawa Coupling Constants}

Yukawa couplings for quarks and leptons in the third generation can be unified with the gauge couplings at compactification scale in the context of gauge-Higgs unification. The gauge-Yukawa (for top-bottom-tau) unification,

$$
g_{1}=g_{2}=g_{3}=y_{t}=y_{b}=y_{\tau}
$$

is studied in Ref. 15. The solid prediction of those coupling unification is $\tan \beta$. As long as the unification condition, Eq. (6.12), is satisfied within $5 \%$ at unification scale, the $\tan \beta$ is predicted as $\tan \beta=52 \pm 1$ when the supersymmetry threshold corrections for tau mass is within a few percents. The predictions of quark masses depend on the low energy supersymmetry threshold corrections. Inputting the experimental data for the SM fermion masses, $m_{\tau}=1.777 \mathrm{GeV}$ and $\bar{m}_{b}\left(\bar{m}_{b}\right)=4.26 \mathrm{GeV}$ (central values from recent lattice calculation) in $\overline{M S}$ scheme [26], the threshold corrections to the bottom quark mass should be less than several percents. Since the finite corrections to the bottom quark mass is proportional to $\tan \beta$, the threshold corrections,

$$
\delta_{b}^{\text {finite }} \simeq-\frac{\alpha_{3}}{3 \pi} \frac{\mu M_{\tilde{g}} \tan \beta}{m_{\tilde{b}}^{2}}+\frac{\alpha_{t}}{8 \pi} \frac{\mu A_{t} \tan \beta}{m_{\tilde{t}}^{2}}
$$




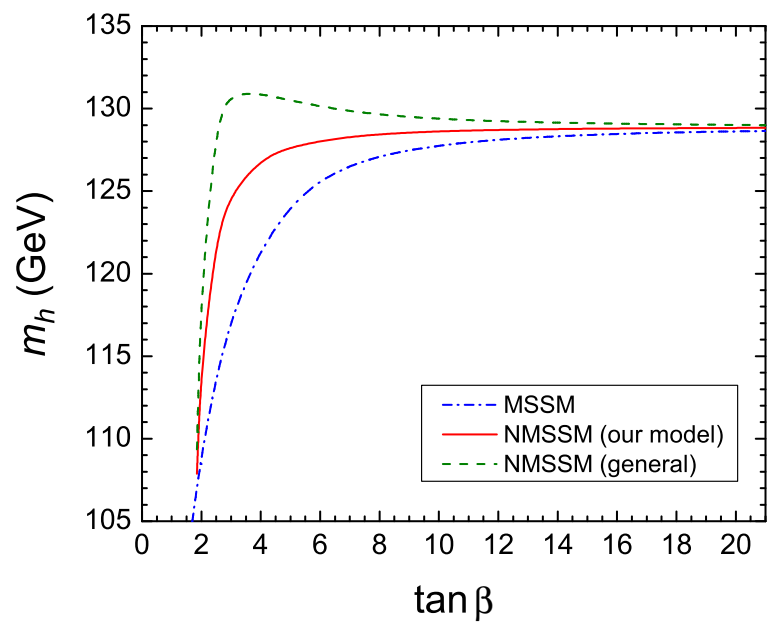

Figure 2: We present the upper bounds on the lightest CP-even Higgs mass in various models: green dashed line corresponds to the general NMSSM case, the blue dash-dotted line corresponds to the MSSM, and the red solid line corresponds to our model.

could be $50 \%$ when the superparticles' masses are about the same. However, there exists the cancellation between gluino mass $M_{\tilde{g}}$ and trilinear scalar coupling for stop $A_{t}$. Such cancellation is needed if we consider the top-bottom-tau Yukawa unification 3. Although the detail prediction of top quark mass depends on the supersymmetric threshold corrections, the top quark mass prediction is in good agreement with experiment.

We also present the evaluation of RGEs for the gauge couplings, Yukawa couplings for the third family, and Higgs trilinear couplings $\kappa$ and $\lambda$ with unification condition at GUT scale in Fig. 3. In those figures, we include the standard supersymmetric threshold corrections at low energy by taking a single scale $M_{S U S Y}=M_{Z}$ [29]. Fig. 3(a) shows the coupling unification in the up-type partial unification. In this case, the figure is shown for small $\tan \beta(\tan \beta \simeq$ $2-15)$ and then the bottom and tau Yukawa couplings are small. The neutrino Dirac Yukawa coupling evolution is also included above the right-handed neutrino Majorana mass scale, which is assumed to be $10^{14} \mathrm{GeV}$. In Fig. 3(b), the down-type partial unification is shown. In this case, $\tan \beta$ is large $(\tan \beta \simeq 51$ ), and the top and bottom Yukawa couplings are comparable.

Because the RGE for top quark Yukawa coupling, $\alpha_{t}$, is given in one-loop (including Diracneutrino Yukawa coupling),

$$
\frac{d \alpha_{t}}{d t}=\frac{\alpha_{t}}{2 \pi}\left(6 \alpha_{t}+\alpha_{b}+\alpha_{\nu_{\tau}}+\alpha_{\lambda}-\frac{13}{15} \alpha_{1}-3 \alpha_{2}-\frac{16}{3} \alpha_{3}\right),
$$

more coupling unification predicts the less top quark mass. We show the prediction of top quark mass in Fig. 4. In the figure, we assume that the couplings $\left(g_{1}, g_{2}\right.$, and $y_{t}$ in the MSSM, and $g_{1}, g_{2}, y_{t}, \lambda$, and $\kappa$ in the NMSSM) in $\overline{D R}$ scheme are unified at the GUT scale. We 

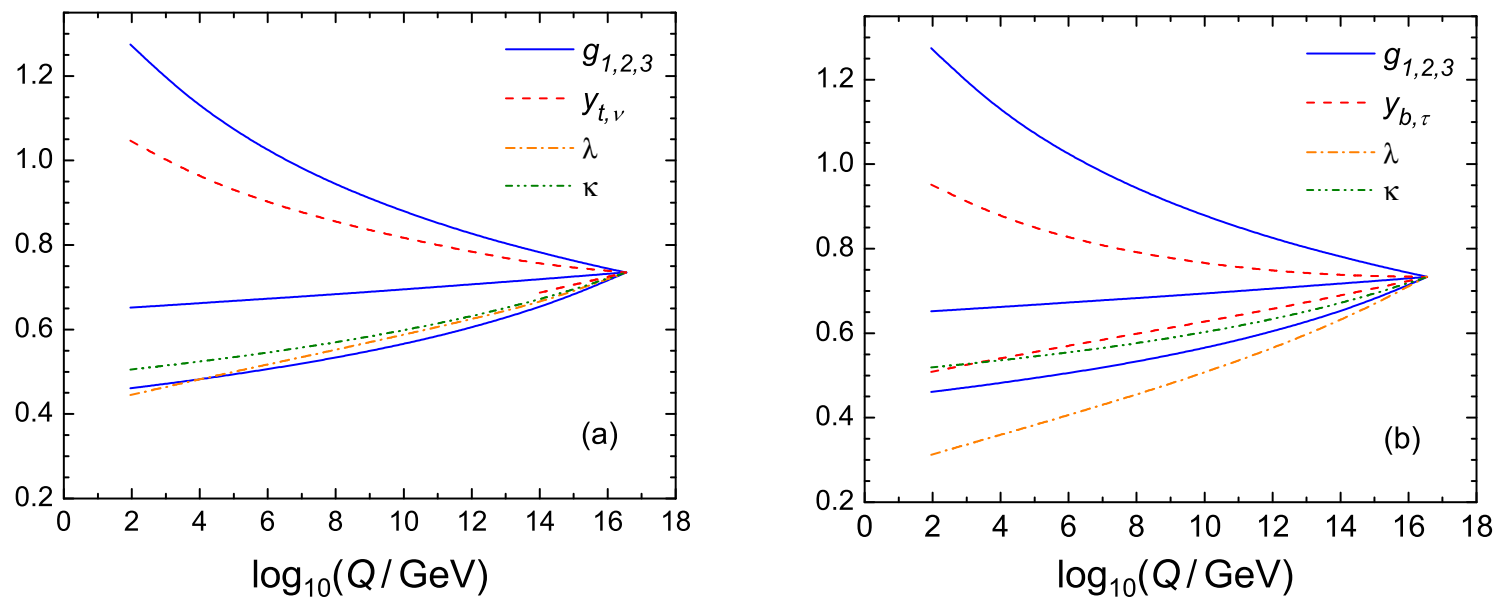

Figure 3: The unification of the gauge $\left(g_{i}\right)$ and Yukawa $\left(y_{f}\right)$ and Higgs $(\lambda$ and $\kappa)$ couplings. Figure (a) shows up-type partial Yukawa unification, and figure (b) shows the down-type one.

use $\alpha_{3}\left(M_{Z}\right)=0.1187$ in $\overline{M S}$ scheme [26]. The top quark mass prediction in the NMSSM (blue solid line) is less than that in the MSSM (red dashed line). The cut in the low $\tan \beta$ region in the figure is just coming from $\sin \beta$ factor in the top quark mass. For large $\tan \beta$ $(\simeq 50)$, the bottom and tau Yukawa couplings can also be unified (neglecting the possible large finite corrections to the bottom quark mass). In this figure, we do not assume the bottomtau unification at GUT scale, but we use the experimental data for the SM fermion masses, $m_{\tau}=1.777 \mathrm{GeV}$ and $\bar{m}_{b}\left(\bar{m}_{b}\right)=4.26 \pm 0.30 \mathrm{GeV}$ in $\overline{M S}$ scheme. In the large $\tan \beta$ region, the bottom quark threshold corrections are sensitive to the predicted top quark mass. In the figure, the possible large supersymmetry threshold corrections for top quark mass are neglected. When the supersymmetry mass parameters are set to be equal to a single scale $M_{S U S Y}$, the top quark mass prediction is insensitive to the $M_{S U S Y}$ (when $M_{S U S Y} \gtrsim 200 \mathrm{GeV}$ ). However, the decoupling type threshold corrections (squarks for only the first and second generations are heavy) may give $1-2 \%$ level corrections to top quark mass. Thus, the top quark mass prediction may have the uncertainties around $\pm 3 \mathrm{GeV}$ due to the threshold corrections when the superpartners are heavy.

Without $\lambda$ coupling unification, the top quark mass prediction is larger than experimental value for the middle range of $\tan \beta$. In this case, the small $\tan \beta(\lesssim 3)$ is disfavored due to the lightest Higgs mass upper bound, while the large $\tan \beta(\simeq 50)$, i.e., the gauge and top-bottomtau Yukawa unification, is favored for the experimental value of the top quark pole mass. On the other hand, in the case that $\lambda$ coupling is also unified to the gauge and top quark Yukawa couplings $\tan \beta \sim 5$ can be a solution (In this case, the lightest Higgs mass upper bound is around $128 \mathrm{GeV}$.). 


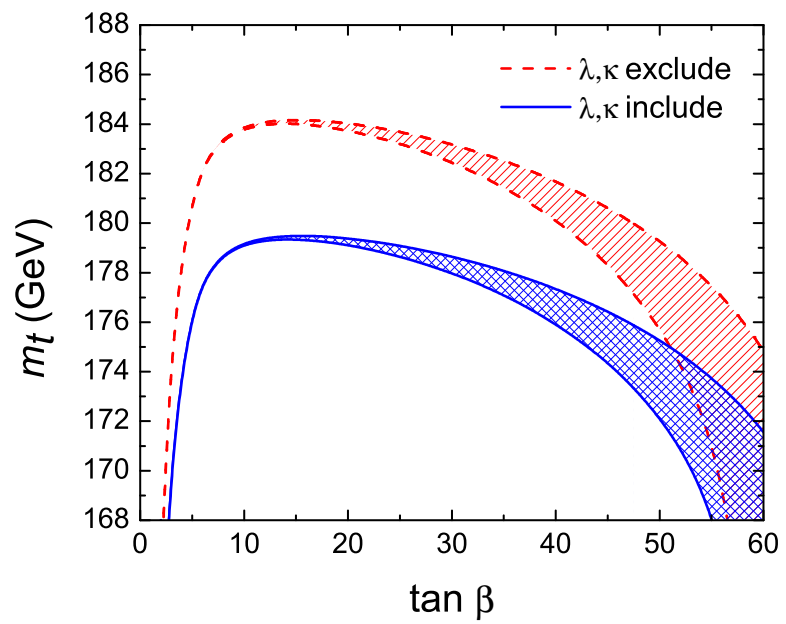

Figure 4: Top quark mass prediction is given as the function of $\tan \beta$. The red dashed lines are for the MSSM with unification of gauge and top quark Yukawa couplings. The blue solid lines are given as the NMSSM with gauge, top quark Yukawa, and Higgs coupling unification. For large $\tan \beta$, the top quark mass prediction is sensitive to the input bottom quark running mass. The shaded areas are shown for the bottom quark mass in the range, $\bar{m}_{b}\left(\bar{m}_{b}\right)=4.26 \pm 0.30$ $\mathrm{GeV}$. The bottom and tau Yukawa couplings can also be unified around $\tan \beta \simeq 50$.

Note that the $\kappa$ coupling unification is less important for the top quark mass prediction since the $\kappa$ gives only two-loop order corrections to the top quark Yukawa coupling. The tau neutrino Dirac Yukawa coupling is also less important since the top quark Yukawa prediction

at low energy is insensitive to the GUT scale corrections, $\delta_{t}^{\mathrm{GUT}} \simeq y_{\nu_{\tau}}^{2} /\left(16 \pi^{2}\right) \ln \left(M_{\mathrm{GUT}} / M_{R}\right)$, where $M_{R}$ is the right-handed neutrino Majorana mass.

If we consider the gauge and only down-type Yukawa (bottom-tau) coupling unification, the top quark mass is just an input parameter. Since the top quark mass in the top-bottom-tau Yukawa unification is in good agreement with the experimental data as an input parameter, such an only down-type Yukawa and gauge coupling unification does not predict brand new physics. In such a case, $\tan \beta$ is predicted around 50 in the same way as the top-bottom-tau Yukawa and gauge coupling unification. Conceptually, the up-type partial unification is very good since it explains why the top quark mass is the heaviest among the SM fermions naturally.

\section{Discussions and Conclusions}

We have studied various possible coupling unifications in the gauge-Higgs unification and gaugeHiggs-matter unification. The gauge-Higgs unification in higher-dimensional supersymmetric models naturally leads to the unification of the gauge and Yukawa couplings. In fact, the 
gauge and Yukawa coupling unification is naturally realized when the grand unification is considered in higher-dimensional supersymmetric models. One can also consider the gaugeHiggs unification in the context of the NMSSM. The trilinear coupling $\lambda S H_{u} H_{d}$, which produces the $\mu$-term, can naturally be generated from the bulk interaction in the simple choice of bulk gauge symmetries. When large enough bulk gauge symmetries are considered, the singlet trilinear coupling, $\kappa S S_{1} S_{2}$, can also be included in the bulk interaction. The $\lambda$ and $\kappa$ couplings at low energy can be calculated by RGE evolution, and thus we expect that the prediction will be tested in the future collider experiments.

We constructed the $S U(8)$ and $S U(9)$ models, which can realize the unification of the NMSSM Higgs trilinear couplings, Yukawa couplings, and gauge couplings. We can select which Yukawa couplings (up-type or down-type) are unified with the gauge and Higgs trilinear couplings by the choice of bulk gauge symmetries and orbifold boundary conditions. The uptype partial unification is very attractive since it naturally explains why the top quark is the heaviest fermion. The predictions can be in very good agreement with experimental observation of the SM fermion masses. Among them, there are two interesting regions allowed by the top quark mass. One is the top-bottom-tau and gauge unification which predict $\tan \beta=52 \pm 1$. In this case, the bottom quark finite corrections should be at several percent level, and thus the supersymmetry breaking parameters should be adjusted. Another one is the $\lambda$ in the NMSSM, the top Yukawa and gauge coupling unification. In the latter case, $\tan \beta$ can be around less than 5 in compatible with the current experimental lightest neutral Higgs mass upper bound. The lightest Higgs mass upper bound is around $128 \mathrm{GeV}$ when the supersymmetry breaking mass scale is $1 \mathrm{TeV}$, and thus the models will be tested at the upcoming Large Hadron Collider.

\section{Acknowledgments}

We would like to thank K. Tobe for useful discussions. This research was supported in part by the National Science Foundation under Grants No. PHY-0098791 (IG) and No. PHY-0070928 (TL), by the Natural Sciences and Engineering Research Council of Canada (YM), and by the Department of Energy Grants DE-FG02-04ER46140 and DE-FG02-04ER41306 (SN).

\section{A Gauge-Higgs Unification in 6D Supersymmetric Mod- els on $T^{2} / Z_{6}$ Orbifold}

We introduce the gauge-Higgs unification in $6 \mathrm{D}$ supersymmetric models. We consider the $6 \mathrm{D}$ space-time which can be factorized into a product of the ordinary 4D Minkowski space-time $M^{4}$, and the torus $T^{2}$. The corresponding coordinates for the space-time are $x^{\mu}(\mu=0,1,2,3)$, 
$x^{5}$ and $x^{6}$. The radii for the circles along the $x^{5}$ and $x^{6}$ directions are $R_{1}$ and $R_{2}$, respectively. We define the complex coordinate

$$
z \equiv \frac{1}{2}\left(x^{5}+i x^{6}\right) .
$$

In the complex coordinate, the torus $T^{2}$ can be defined by $C^{1}$ moduloing the equivalent classes:

$$
z \sim z+\pi R_{1}, \quad z \sim z+\pi R_{2} e^{\mathrm{i} \theta} .
$$

To define $T^{2} / Z_{6}$ orbifold, we require that $R_{1}=R_{2} \equiv R$ and $\theta=\pi / 3$. The $T^{2} / Z_{6}$ orbifold is obtained from $T^{2}$ by moduloing the equivalent class

$$
\Gamma_{T}: z \sim \omega z,
$$

where $\omega=e^{\mathrm{i} \pi / 3}$. There is one $Z_{6}$ fixed point $z=0$, two $Z_{3}$ fixed points: $z=\pi R e^{\mathrm{i} \pi / 6} / \sqrt{3}$ and $z=2 \pi R e^{\mathrm{i} \pi / 6} / \sqrt{3}$, and three $Z_{2}$ fixed points: $z=\sqrt{3} \pi R e^{\mathrm{i} \pi / 6} / 2, z=\pi R / 2$ and $z=\pi R e^{\mathrm{i} \pi / 3} / 2$.

The $\mathcal{N}=(1,1)$ supersymmetry in 6D has 16 supercharges and corresponds to the $\mathcal{N}=4$ supersymmetry in $4 \mathrm{D}$, thus, only the gauge multiplet can be introduced in the bulk. This multiplet can be decomposed under the $4 \mathrm{D} \mathcal{N}=1$ supersymmetry into a vector multiplet $V$ and three chiral multiplets $\Sigma_{1}, \Sigma_{2}$, and $\Sigma_{3}$ in the adjoint representation, where the fifth and sixth components of the gauge field, $A_{5}$ and $A_{6}$, are contained in the lowest component of $\Sigma_{1}$.

We write down the bulk action in the Wess-Zumino gauge and 4D $\mathcal{N}=1$ supersymmetry language [25],

$$
\begin{aligned}
\mathcal{S}= & \int d^{6} x\left\{\operatorname{Tr}\left[\int d^{2} \theta\left(\frac{1}{4 k g^{2}} \mathcal{W}^{\alpha} \mathcal{W}_{\alpha}+\frac{1}{k g^{2}}\left(\Sigma_{3} \partial \Sigma_{2}-\sqrt{2} \Sigma_{1}\left[\Sigma_{2}, \Sigma_{3}\right]\right)\right)+\text { H.C. }\right]\right. \\
& +\int d^{4} \theta \frac{1}{k g^{2}} \operatorname{Tr}\left[\left(\frac{1}{\sqrt{2}} \partial_{z}^{\dagger}+\Sigma_{1}^{\dagger}\right) e^{-2 V}\left(-\frac{1}{\sqrt{2}} \partial_{z}+\Sigma_{1}\right) e^{2 V}+\frac{1}{4} \partial_{z}^{\dagger} e^{-2 V} \partial_{z} e^{2 V}\right] \\
& \left.+\int d^{4} \theta \frac{1}{k g^{2}} \operatorname{Tr}\left[\Sigma_{2}^{\dagger} e^{-2 V} \Sigma_{2} e^{2 V}+\Sigma_{3}^{\dagger} e^{-2 V} \Sigma_{3} e^{2 V}\right]\right\} .
\end{aligned}
$$

The above action is invariant under the following orbifold transformation conditions:

$$
\begin{aligned}
V\left(x^{\mu}, \omega z, \omega^{-1} \bar{z}\right) & =R V\left(x^{\mu}, z, \bar{z}\right) R^{-1}, \\
\Sigma_{1}\left(x^{\mu}, \omega z, \omega^{-1} \bar{z}\right) & =\omega^{-1} R \Sigma_{1}\left(x^{\mu}, z, \bar{z}\right) R^{-1}, \\
\Sigma_{2}\left(x^{\mu}, \omega z, \omega^{-1} \bar{z}\right) & =\omega^{-1-m} R \Sigma_{2}\left(x^{\mu}, z, \bar{z}\right) R^{-1}, \\
\Sigma_{3}\left(x^{\mu}, \omega z, \omega^{-1} \bar{z}\right) & =\omega^{2+m} R \Sigma_{3}\left(x^{\mu}, z, \bar{z}\right) R^{-1},
\end{aligned}
$$

where we introduce the non-trivial action on the gauge space, $R$, which can break the bulk gauge group $G$ down to $H$ on the $Z_{6}$ fixed points. To keep the $4 \mathrm{D} \mathcal{N}=1$ supersymmetry, we need $m=0$ or $m=1$ [24]. 
Under the orbifold conditions, any bulk fields have $Z_{6}$ charges, such as

$$
\phi\left(x^{\mu}, \omega z, \omega^{-1} \bar{z}\right)=\omega^{a} \phi\left(x^{\mu}, z, \bar{z}\right) .
$$

The bulk field with $a \neq 0$ vanishes at the fixed point, and then does not have massless mode. On the other hand, the bulk field with $a=0$ contains zero-mode in 4D. By introducing non-trivial $R$, the bulk vector multiplet is decomposed as the following in the matrix presentation:

$$
V=\left(\begin{array}{cccc}
V^{(1)} & V^{(12)} & \cdots & V^{(1 n)} \\
V^{(21)} & V^{(2)} & \cdots & V^{(2 n)} \\
\vdots & \vdots & \ddots & \vdots \\
V^{(n 1)} & V^{(n 2)} & \cdots & V^{(n)}
\end{array}\right), \quad \Sigma_{i}=\left(\begin{array}{cccc}
\Sigma_{i}^{(1)} & \Sigma_{i}^{(12)} & \cdots & \Sigma_{i}^{(1 n)} \\
\Sigma_{i}^{(21)} & \Sigma_{i}^{(2)} & \cdots & \Sigma_{i}^{(2 n)} \\
\vdots & \vdots & \ddots & \vdots \\
\Sigma_{i}^{(n 1)} & \Sigma_{i}^{(n 2)} & \cdots & \Sigma_{i}^{(n)}
\end{array}\right),
$$

where each decomposed component can have different $Z_{6}$ charges. By definition, $V^{(i)}$ 's have charge 0 , and correspond to vector multiplet for gauge group $H$, while $V^{(i j)}$ 's, which correspond to $G / H$, do not have zero-modes. On the other hand, due to the conditions in Eqs. A.6.8), $\Sigma_{i}^{(i)}$ s do not contain massless modes unless $m=+1,-2$, and some of the $\Sigma_{i}^{(i j)}$ can have massless modes. The off-diagonal components in the decomposition correspond to the bifundamental representations under $H$ when $G=S U(N)$, and then the zero-modes in $\Sigma_{i}^{(i j)}$ can be identified as the Higgs fields. Thus, the gauge and Higgs unification is realized naturally. Furthermore, the bulk action, Eq. (A.4), includes the trilinear couplings for the massless modes of $\Sigma_{i}^{(i j)}$,

$$
\operatorname{Tr} \Sigma_{1}\left[\Sigma_{2}, \Sigma_{3}\right]=\Sigma_{1}^{(i j)}\left(\Sigma_{2}^{(j k)} \Sigma_{3}^{(k i)}-\Sigma_{3}^{(j k)} \Sigma_{2}^{(k i)}\right) .
$$

Therefore, the trilinear coupling constants for the normalized scalar fields, $\sqrt{2} \Sigma_{i}^{(i j)}$, are the same as the gauge coupling constants in the conventional definition $(k=1 / 2)$.

\section{References}

[1] U. Amaldi, W. de Boer and H. Furstenau, Phys. Lett. B 260, 447 (1991); J. R. Ellis, S. Kelley and D. V. Nanopoulos, Phys. Lett. B 249, 441 (1990); P. Langacker and M. X. Luo, Phys. Rev. D 44, 817 (1991); C. Giunti, C. W. Kim and U. W. Lee, Mod. Phys. Lett. A 6 (1991) 1745.

[2] For recent analyses, S. Komine and M. Yamaguchi, Phys. Rev. D 65, 075013 (2002) hep-ph/0110032; U. Chattopadhyay and P. Nath, Phys. Rev. D 65, 075009 (2002) hep-ph/0110341.

[3] For recent analyses, H. Baer and J. Ferrandis, Phys. Rev. Lett. 87, 211803 (2001) hep-ph/0106352. T. Blazek, R. Dermisek and S. Raby, Phys. Rev. Lett. 88, 111804 
(2002) hep-ph/0107097; Phys. Rev. D 65, 115004 (2002) hep-ph/0201081; K. Tobe and J. D. Wells, Nucl. Phys. B 663, 123 (2003) hep-ph/0301015.

[4] T. Fukuyama and N. Okada, JHEP 0211, 011 (2002) hep-ph/0205066; B. Bajc, G. Senjanovic and F. Vissani, Phys. Rev. Lett. 90, 051802 (2003) hep-ph/0210207; H. S. Goh, R. N. Mohapatra and S. P. Ng, Phys. Lett. B 570, 215 (2003) hep-ph/0303055; B. Dutta, Y. Mimura and R. N. Mohapatra, Phys. Rev. Lett. 94, 091804 (2005) hep-ph/0412105.

[5] N. S. Manton, Nucl. Phys. B 158, 141 (1979); D. B. Fairlie, J. Phys. G 5, L55 (1979); Phys. Lett. B 82, 97 (1979); P. Forgacs and N. S. Manton, Commun. Math. Phys. 72, 15 (1980); G. Chapline and R. Slansky, Nucl. Phys. B 209, 461 (1982); S. Randjbar-Daemi, A. Salam and J. Strathdee, Nucl. Phys. B 214, 491 (1983); I. Antoniadis, Phys. Lett. B 246, 377 (1990); N. V. Krasnikov, Phys. Lett. B 273, 246 (1991); D. Kapetanakis and G. Zoupanos, Phys. Rept. 219, 1 (1992); I. Antoniadis and K. Benakli, Phys. Lett. B 326, 69 (1994) hep-th/9310151; H. Hatanaka, T. Inami and C. S. Lim, Mod. Phys. Lett. A 13, 2601 (1998) hep-th/9805067.

[6] Y. Hosotani, Phys. Lett. B 126, 309 (1983); Phys. Lett. B 129, 193 (1983); Phys. Rev. D 29, 731 (1984); Annals Phys. 190, 233 (1989).

[7] G. R. Dvali, S. Randjbar-Daemi and R. Tabbash, Phys. Rev. D 65, 064021 (2002) hep-ph/0102307; N. Arkani-Hamed, A. G. Cohen and H. Georgi, Phys. Lett. B 513, 232 (2001) hep-ph/0105239; L. J. Hall, Y. Nomura and D. R. Smith, Nucl. Phys. B 639, 307 (2002) hep-ph/0107331; I. Antoniadis, K. Benakli and M. Quiros, New J. Phys. 3, 20 (2001) hep-th/0108005; M. Kubo, C. S. Lim and H. Yamashita, Mod. Phys. Lett. A 17, 2249 (2002) hep-ph/0111327; C. Csaki, C. Grojean and H. Murayama, Phys. Rev. D 67, 085012 (2003) hep-ph/0210133; C. A. Scrucca, M. Serone and L. Silvestrini, Nucl. Phys. B 669, 128 (2003) hep-ph/0304220; N. Haba, Y. Hosotani, Y. Kawamura and T. Yamashita, Phys. Rev. D 70, 015010 (2004) hep-ph/0401183 Y. Hosotani, S. Noda and K. Takenaga, Phys. Lett. B 607, 276 (2005) hep-ph/0410193.

[8] Y. Kawamura, Prog. Theor. Phys. 103, 613 (2000) hep-ph/9902423; ibid. 105, 999 (2001) hep-ph/0012125; ibid. 105, 691 (2001) hep-ph/0012352.

[9] Y. Mimura and S. Nandi, Phys. Lett. B 538, 406 (2002) hep-ph/0203126; I. Gogoladze, Y. Mimura and S. Nandi, Phys. Lett. B 554, 81 (2003) hep-ph/0210320.

[10] G. Altarelli and F. Feruglio, Phys. Lett. B 511, 257 (2001) hep-ph/0102301; A. B. Kobakhidze, Phys. Lett. B 514, 131 (2001) hep-ph/0102323; L. J. Hall and Y. No- 
mura, Phys. Rev. D 64, 055003 (2001) hep-ph/0103125; Phys. Rev. D 65, 125012 (2002) hep-ph/0111068; A. Hebecker and J. March-Russell, Nucl. Phys. B 613, 3 (2001) hep-ph/0106166; T. Li, Phys. Lett. B 520, 377 (2001) hep-th/0107136; Nucl. Phys. B 619, 75 (2001) hep-ph/0108120; T. Asaka, W. Buchmuller and L. Covi, Phys. Lett. B 523, 199 (2001) hep-ph/0108021.

[11] G. Burdman and Y. Nomura, Nucl. Phys. B 656, 3 (2003) hep-ph/0210257; N. Haba and Y. Shimizu, Phys. Rev. D 67, 095001 (2003) hep-ph/0212166.

[12] I. Gogoladze, Y. Mimura and S. Nandi, Phys. Lett. B 560, 204 (2003) hep-ph/0301014.

[13] I. Gogoladze, Y. Mimura and S. Nandi, Phys. Lett. B 562, 307 (2003) hep-ph/0302176; Phys. Rev. Lett. 91, 141801 (2003) hep-ph/0304118.

[14] I. Gogoladze, Y. Mimura and S. Nandi, Phys. Rev. D 69, 075006 (2004) hep-ph/0311127.

[15] I. Gogoladze, Y. Mimura, S. Nandi and K. Tobe, Phys. Lett. B 575, 66 (2003) hep-ph/0307397.

[16] G. Lazarides and Q. Shafi, Phys. Rev. D 58, 071702 (1998) hep-ph/9803397; K. Kurosawa, N. Maru and T. Yanagida, Phys. Lett. B 512, 203 (2001) hep-ph/0105136; K. S. Babu, I. Gogoladze and K. Wang, Nucl. Phys. B 660, 322 (2003) hep-ph/0212245; Phys. Lett. B 560, 214 (2003) hep-ph/0212339.

[17] G. F. Giudice and A. Masiero, Phys. Lett. B 206, 480 (1988).

[18] P. Fayet, Nucl. Phys. B 90, 104 (1975); H. P. Nilles, M. Srednicki and D. Wyler, Phys. Lett. B 120, 346 (1983); J. M. Frere, D. R. T. Jones and S. Raby, Nucl. Phys. B 222, 11 (1983); J. P. Derendinger and C. A. Savoy, Nucl. Phys. B 237, 307 (1984).

[19] I. Gogoladze, T. Li, Y. Mimura and S. Nandi, hep-ph/0501264

[20] S. Dimopoulos and D. E. Kaplan, Phys. Lett. B 531, 127 (2002) hep-ph/0201148.

[21] Q. Shafi and Z. Tavartkiladze, Phys. Rev. D 66, 115002 (2002); N. Cosme and J. M. Frere, Phys. Rev. D 69, 036003 (2004) hep-ph/0303037.

[22] J. C. Pati and A. Salam, Phys. Rev. D 10, 275 (1974).

[23] S. A. Abel, S. Sarkar and P. L. White, Nucl. Phys. B 454, 663 (1995) hep-ph/9506359;

C. Panagiotakopoulos and K. Tamvakis, Phys. Lett. B 446, 224 (1999) hep-ph/9809475. 
[24] T. Li, JHEP 0403, 040 (2004) hep-ph/0309199.

[25] N. Marcus, A. Sagnotti and W. Siegel, Nucl. Phys. B 224, 159 (1983); N. Arkani-Hamed, T. Gregoire and J. Wacker, JHEP 0203, 055 (2002) hep-th/0101233.

[26] S. Eidelman et al. [Particle Data Group], Phys. Lett. B 592, 1 (2004).

[27] D. R. T. Jones, Phys. Rev. D 25, 581 (1982); M. E. Machacek and M. T. Vaughn, Nucl. Phys. B 236, 221 (1984). S. P. Martin and M. T. Vaughn, Phys. Rev. D 50, 2282 (1994) hep-ph/9311340; S. F. King and P. L. White, Phys. Rev. D 52, 4183 (1995) hep-ph/9505326.

[28] Y. Okada, M. Yamaguchi and T. Yanagida, Prog. Theor. Phys. 85, 1 (1991); J. R. Ellis, G. Ridolfi and F. Zwirner, Phys. Lett. B 257, 83 (1991); H. E. Haber and R. Hempfling, Phys. Rev. Lett. 66, 1815 (1991); M. Carena, J. R. Espinosa, M. Quiros and C. E. M. Wagner, Phys. Lett. B 355, 209 (1995). hep-ph/9504316.

[29] P. Langacker and N. Polonsky, Phys. Rev. D 47, 4028 (1993) hep-ph/9210235. M. Carena, S. Pokorski and C. E. M. Wagner, Nucl. Phys. B 406, 59 (1993) hep-ph/9303202. 